\title{
A Novel Cache Architecture and Placement Framework for Packet Forwarding Engines
}

\author{
Kaushik Rajan and Ramaswamy Govindarajan, Senior Member, IEEE
}

\begin{abstract}
Packet forwarding is a memory-intensive application requiring multiple accesses through a trie structure. With the requirement to process packets at line rates, high-performance routers need to forward millions of packets every second with each packet needing up to seven memory accesses. Earlier work shows that a single cache for the nodes of a trie can reduce the number of external memory accesses. It is observed that the locality characteristics of the level-one nodes of a trie are significantly different from those of lower level nodes. Hence, we propose a heterogeneously segmented cache architecture (HSCA) which uses separate caches for level-one and lower level nodes, each with carefully chosen sizes. Besides reducing misses, segmenting the cache allows us to focus on optimizing the more frequently accessed level-one node segment. We find that due to the nonuniform distribution of nodes among cache sets, the level-one nodes cache is susceptible to high conflict misses. We reduce conflict misses by introducing a novel two-level mapping-based cache placement framework. We also propose an elegant way to fit the modified placement function into the cache organization with minimal increase in access time. Further, we propose an attribute preserving trace generation methodology which emulates real traces and can generate traces with varying locality. Performance results reveal that our HSCA scheme results in a 32 percent speedup in average memory access time over a unified nodes cache. Also, HSCA outperforms IHARC, a cache for lookup results, with as high as a 10-fold speedup in average memory access time. Two-level mapping further enhances the performance of the base HSCA by up to 13 percent leading to an overall improvement of up to 40 percent over the unified scheme.
\end{abstract}

Index Terms-Special-purpose and application-based systems, design, performance, experimentation, cache architectures, network processors, synthetic trace generation, trace driven simulation.

\section{INTRODUCTION}

$\mathrm{T}$ HE ever increasing demand for network bandwidth and the need to support sophisticated streaming applications entail that routers process packets at higher line rates. Due to the memory-intensive nature of packet forwarding, modern day routers are constrained by the performance of the memory system rather than the processing capability. One of the key functionalities of routers is to forward incoming packets through an appropriate output port. This involves looking in a table of prefixes, finding the longest prefix match (LPM) for the destination IP-address, and using the corresponding output port.

Most algorithms used for LPM make use of a trie data structure to store the contents of the routing table [4], [29]. Even with efficient lookup algorithms, a single internet packet (IP)-lookup incurs a significant number of memory accesses. For example, in the worst case, the LC-trie algorithm [23] requires seven memory accesses and the Lulea algorithm [3] requires as many as 12 memory accesses. With the requirement to process packets at wire speeds (10 Gbps or higher), a router has to process more than 20 million packets every second. Under such requirements supporting, IP-lookup stresses the memory system greatly.

Routers typically use a multithreaded multiprocessor architecture to hide memory latencies. Caching is a technique

- K. Rajan is with Microsoft Research, Sadashiva Nagar, Bangalore, 560080, India. E-mail: krajan@microsoft.com.

- R. Govindarajan is with SERC, Indian Institute of Science, Bangalore, 560012, India. E-mail: govind@serc.iisc.ernet.in.

Manuscript received 12 July 2007; revised 7 Aug. 2008; accepted 20 Nov. 2008; published online 9 Jan. 2009.

Recommended for acceptance by A. Gonzalez.

For information on obtaining reprints of this article, please send e-mail to: tc@computer.org, and reference IEEECS Log Number TC-2007-07-0314. Digital Object Identifier no. 10.1109/TC.2009.18. orthogonal to multithreading for reducing memory access overhead in network applications. While multithreading hides memory latency and improves processor utilization, caching reduces the number of accesses that go to memory and reduces average memory access time. It improves performance by reducing the contention for memory, preventing saturation of memory bandwidth, and reducing the number of thread context switches. Earlier work suggests that multithreading alone is not sufficient to ease the memory bottleneck. Caching can further improve the throughput by a large margin [2], [21].

The locality characteristics of IP-address traces have been well studied [7], [15]. Such traces tend to have no spatial locality but do tend to exhibit temporal locality. The temporal locality characteristics vary based on the location of the router in consideration. It is observed that at edge routers, the IP-addresses tend to exhibit much greater temporal locality than at core routers. For example, traces observed at the edge routers tend to have less than 1 percent of unique addresses in them, whereas the core router traces typically contain 10 percent or greater unique addresses [22]. In order to evaluate the design considerations for routers, we develop a parameterized synthetic trace generation methodology [25], [26]. Traces generated by our attribute preserving trace generator obey the prefix length distribution of real traces [22] Further, by varying the parameters, we generate traces which correspond to edge or core routers.

Various proposals have been made to use caches to speedup the lookup algorithm [2], [5], [6], [36]. However, in spite of using a cache, the number of external memory accesses incurred can be as high as 75 percent of all requests [2]. Thus, any reduction in misses should significantly improve the average case performance of the router. We propose a novel cache organization for routers that is fine tuned to make use of the high-level properties of the 


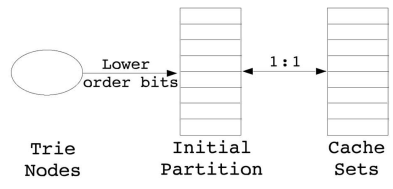

(a)

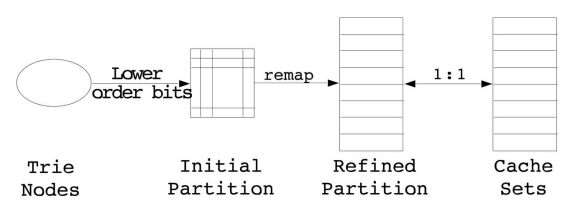

(b)

Fig. 1. Mapping from nodes to sets. (a) Conventional mapping. (b) Twolevel mapping.

algorithms used for forwarding. In doing so, we make use of the observation that routing table entries change infrequently, and hence, the working set that will be active in the near future is known in advance. The challenge is to fit this working set elegantly in a cache. Also, care needs to be taken to ensure that the proposed solution is generic enough to be applicable to any distribution of prefixes in the routing table. We propose simple modifications to the cache organization to accomplish the above.

Earlier work shows that the use of a single unified cache to store all-level nodes of a forwarding trie can improve the throughput of routers [2]. Typically, in any trie used for forwarding the root node has a large number of children $\left(2^{16}\right)$, while other nodes have relatively smaller number of children. Every lookup involves at least an access to a LevelOne (LO) node (direct children of root) and may access lower trie nodes. As a consequence, the locality characteristics of LO nodes are significantly different from those of Lower Level (LL) nodes (nodes other than LO nodes and root node).

We propose a Heterogeneous Segmented Cache Architecture (HSCA) [25] tailor-made to suit the packet forwarding application and exploit the locality characteristics exhibited by the application. HSCA uses separate caches for LO and LL nodes with sizes chosen based on the number of accesses to each partition. As about 85 percent of the accesses are to LO nodes, we skew the size of LO cache to be eight times the size of LL cache. This difference in sizes makes the cache heterogeneous in nature. Further, this heavy skew toward the LO motivates the need to exploit the LO cache organization in more detail. We optimize the LO cache by introducing a weight-based replacement policy that assigns weights to nodes based on their importance in the trie. We also observe that the usage of a large branching factor $\left(2^{16}\right)$ for the root node [29] introduces a large number of superfluous LO nodes. These are basically LO nodes which get added when a fixed root branching factor is used but do not contain any information, and hence, are never accessed (Section 7 provides an example). For the routing tables we use, a branching factor of $2^{16}$ results in as many as 75 percent superfluous LO nodes. As these superfluous nodes are interspersed with useful nodes, using a conventional placement function (indexing with lower order bits) for the LO cache may not uniformly distribute the useful nodes among the sets. Hence, a conventional placement function may result in more conflict misses.

The mapping achieved by conventional placement functions is depicted in Fig. 1a. In these mappings, the set of useful LO nodes are partitioned based on the lower order $\log m$ bits into $m$ partitions. These $m$ partitions are then mapped one-to-one to $m$ cache sets. Both the partitioning and the mapping of partitions to sets is rigid. While this rigidity in the partitioning and mapping helps to achieve efficient direct indexing and hence low hit-latency, we observe that it results in an uneven distribution of useful LO nodes among partitions.

In contrast, we propose a two-level mapping framework [26] which achieves the desired flexibility to reduce conflict misses without significantly impacting access latency. The framework (refer to Fig. 1b) maintains a rigid partitioning of useful LO nodes into initial partitions (IP), but reorganizes the nodes in the IPs into more evenly populated refined partitions (RPs) through intelligent cache index selection. The RPs are then rigidly (one-to-one) mapped to cache sets. By introducing an additional layer of flexible mapping between IP and RP, a better distribution of LO nodes to sets can be achieved.

We further propose an elegant way to incorporate twolevel mapping into the existing cache organization without significantly affecting the access time. We propose three schemes to intelligently remap the nodes in IPs into RPs namely FLEX, IMAP, and V-ASSOC. In each of these schemes, the remapping used for one IP can be different from that of another. As routing tables change infrequently, these remap functions can be computed offline before a routing table is put to use. This provides us with a flexibility to adapt the node to set mapping to a given routing table.

The performance of HSCA is compared with two contemporary caching schemes, namely the single unified cache [2] and the Intelligent Host Address Range Caching (IHARC) [6]. Performance evaluation reveals that the base HSCA scheme gives us up to 25 percent reduction in misses over the unified scheme resulting in 32 percent memory access speedup. Relative to IHARC, HSCA results in up to 90 percent savings in number of misses for edge router traces leading to a 10 -fold speedup in memory access time. For core router traces, the weight-based replacement further enhances performance leading to up to 25 percent lesser misses than the unified cache. Further, when two-level mapping is introduced, the number of misses of the LO cache reduce by 16 percent resulting in an overall memory access speedup of up to 40 percent over the unified cache. In comparison, XORbased placement achieves at best a 3 percent reduction in $\mathrm{LO}$ misses. Further, we perform a detailed timing analysis based on HSPICE simulations to show that two-level mapping does not impact the cache access time.

In Section 2, we provide a background on IP-forwarding. In Section 3, we discuss relevant related work. Section 4 describes our attribute preserving trace generation methodology. In Section 5, we introduce HSCA and elaborate on our weight-based replacement policy. Section 6 reports the experimental results and compares HSCA with related work. Section 7 motivates the need for two-level mapping. In Section 8, we present the cache organization required for various remapping schemes. The performance of two-level mapping-based schemes is reported in Section 9 and we conclude in Section 10.

\section{BACKGROUND}

In order to facilitate Longest Prefix Matching, routers typically represent the routing table information in the form of a trie [4], [29]. In this paper, we only focus on forwarding using tries as this is the most abundantly used data structure for forwarding [29]. Consider the routing table in Fig. 2a. This table can be represented by a simple 


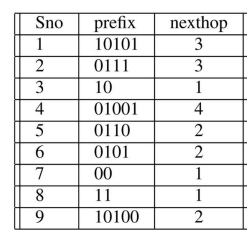

(a)

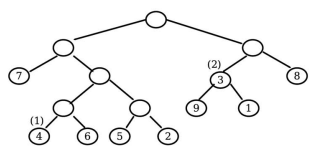

(c)

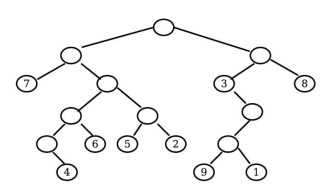

(b)

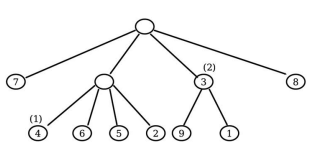

(d)
Fig. 2. LC tries for forwarding. (a) Routing table. (b) Binary trie. (c) Path compressed trie. (d) LC trie.

binary trie as shown in Fig. 2b. This trie is traversed using the bits of the IP-address being looked up. The traversal takes one bit at a time and goes to the left (right) child if the bit is 0 (1). The search ends when a leaf node is reached.

A simple binary trie of Fig. $2 b$ has a large number of nodes and a high average depth. The various trie-based schemes use a compact representation of the simple binary trie and differ only in the manner in which they compress the trie. Below we discuss a few commonly used compression schemes. A more detailed discussion can be found elsewhere [4], [29].

Path compression. Nodes which have only one child are removed from the trie. The number of such nodes that have been skipped, the skip value, is stored in a node along the path. While performing the lookup, the skip value information is used to determine which is the next bit to use for traversal. The path compressed form of Fig. $2 b$ is shown in Fig. 2c. This scheme was originally introduced in [20] and is commonly used in many trie-based algorithms including the radix trie in the BSD Linux kernel.

Level compression with prefix expansion. Instead of using one bit at a time, $k$ bits are used at one go to proceed to one of the $2^{k}$ (called the branching factor or bf) children. This would save $k-1$ visits in a binary trie. This requires expanding existing nodes up to the required depth (prefix expansion). As some of the nodes may not exist in the original trie, superfluous nodes might have to be added. A quality metric is usually used to restrict expansion to useful parts of the trie. For example, a dynamic program is used to restrict prefix expansion in [35]. In the LC-trie scheme, a branching factor of $2^{k}$ is used if it produces at most $b f *(1-x)$ superfluous leaves [23]. The level compressed form of Fig. 2c with $x=1$ is shown in Fig. 2 d.

Root branching factor. As routing tables have very few small prefixes most forwarding algorithms use a trie structure with a large number of children for the root node [17], [12], [23], [38]. Typically, a root branching factor of $2^{16}$ is used. Also, as the number of children of the root is so high, using pointers to each of the children is infeasible. Instead, the level-one nodes are stored in an array structure.

An efficiently compressed trie structure used in earlier cache studies [2] is the Level Compressed trie [23], [27]. In an LC-trie, all the above three forms of compression are used. We use this algorithm for all our evaluations as well. The fill factor $x$ is assumed to be 0.5 as it gives the best compression [23].

In an LC trie-based algorithm, after reaching a leaf node, the nexthop information can be obtained from auxiliary tables. Alternatively, as suggested in [27], additional information can be embedded in the trie itself and the auxiliary tables can be completely eliminated. A more detailed discussion on the organization required to do so can be found in Section 6.3.

\section{Related Work}

Due to the highly parallel nature of network applications, the memory subsystem and not the computational resources are the bottleneck [2], [21], [30]. Baer et al. [2] demonstrate that a combination of caching and multithreading would be effective at bridging this performance gap for packet forwarding algorithms. Mudigonda et al. [21] compare a few contemporary schemes for reducing the memory performance bottleneck. These include multithreading, wide-word memories, caching-based solutions, and nonblocking data transfers. The study reveals that at least a combination of multithreading and caching is essential for a balanced NP architecture.

Below we discuss a few recent proposals that address the memory performance gap for architectures tuned to network applications and discuss caching-based solutions in more detail. Sherwood et al. propose to use wide-word accesses and internally pipelined memory architectures [31] to enhance the memory throughput. They suggest ways to tune existing network algorithms to utilize the wide-word parallelism exposed by their architecture. Hasan et al. [13] propose mechanisms to exploit the locality among DRAM accesses. By reordering outstanding accesses to expose row locality and performing locality sensitive buffer allocation, they reduce the average DRAM access time. A virtually pipelined network memory architecture is introduced in [1] that, in the presence of large number of memory banks, ensures predictable latencies for all memory accesses with very high probability. While these schemes try to exploit the parallelism provided by the multibanked organization of memory, we propose to reduce the average memory access time using caching mechanisms. We do not make any assumptions about the parallelism exposed by the memory architecture and explore ways of utilizing inherent locality among accesses to the forwarding data structure. As suggested in [2], [21], a combination of approaches is required to bridge the memory gap.

Packet forwarding is a primary functionality for network processors. A number of caching-based solutions have been proposed to speedup packet forwarding algorithms [2], [5], [6], [18], [28]. Before we discuss these schemes in detail, a few common organizational features of these caches are discussed. First, caches proposed for forwarding accommodate exactly one entry per cache line. This is because of the total lack of spatial correlation between successively accessed prefixes. Second, every time the entries of the routing table in use change, the cache entries become stale and need to be flushed/synchronized. However, to keep the overhead of frequent route updates low, route updates are initially made to a backup (shadow) routing table. This backup table replaces the in-use table much less frequently [4], [17]. As a consequence, the cache need not be flushed on every route update, but can simply be flushed every time the backup routing table is put to use. Attempts at caching for IP-forwarding can be broadly classified into two categories: caching address ranges or prefixes [5], [6] and caching data structures used for IP-lookup [2]. Among prefix caching proposals, an associative ternary cache [28] is organized such that cache lines are ordered based on prefix lengths. The appropriate bits of the incoming address are 
compared with all cached prefixes and longest prefix match is then chosen through a priority encoder. A route prefix cache is a small fully associative cache [18] which stores a mask along with the address to facilitate prefix matching. To ensure longest prefix matching, all subprefixes are marked as noncachable. Further, to reduce the number of noncachable entries, a limited expansion of the tree is performed. The prefix cache is extended with a new replacement policy, mLRU [19]. The proposed policy effectively combines LRU and LFU by ensuring that longer flows are given more weightage over shorter flows.

The IHARC scheme [6] proposes caching of address ranges and their routing decisions. By carefully choosing the bits to index the cache, the number of address ranges mapping to a cache set is minimized. Once the index bits are removed, certain address ranges that are not originally adjacent may become adjacent. If such adjacent ranges share the same routing decision, they are merged together. In IHARC, unlike a conventional cache, there could be entries in the cache matching many addresses.

This slightly complicates the cache logic as the tag match now involves applying a mask to the address tag before doing a comparison. This work is extended by introducing additional splitting bits which facilitate a variable cache set mapping, where partitions to which more number of address ranges map are given more sets [5]. A major drawback of prefix caching schemes (as is evident in the above discussed schemes) is that the requirement of the longest prefix matching complicates the design of the cache. We compare our proposed solution with the IHARC scheme and show significant benefits.

Recent research focuses on caching the data structures used for forwarding. The additional locality in accesses to the nodes of a trie data structure used for forwarding is exploited by using a cache to store the recently accessed nodes of an LC-trie [2]. A single cache is used to store alllevel nodes (henceforth referred to as the unified cache scheme) and uses LRU replacement. Our scheme is different from the above in that it leverages performance by tuning the cache organization to the locality characteristics of nodes at various levels. In this paper, we compare the performance of our proposal with one scheme from each category (IHARC and unified). Performance evaluation reveals that HSCA outperforms both these schemes.

In the context of general purpose computing, XOR-based placement schemes [10] and prime modulo indexing schemes [16] have been proposed to reduce conflict misses. Our proposed two-level mapping scheme can make use of application specific working set information. Performance evaluation indicates that an XOR-based scheme is not very effective in this domain. Lastly, in the context of embedded processors, an intelligent index bit selection method is proposed [9] to reduce conflict misses. Our performance evaluation reveals that a bit selection-based approach is also not very effective for packet forwarding.

\section{Synthetic Trace Generation}

One major difficulty in the performance evaluation of routers and routing algorithms is the lack of publicly available traces that correspond to publicly available routing tables. This difficulty can be overcome by using synthetic traces. However, in order for these evaluations to be useful, the synthetic trace should match the characteristics of real traces. Synthetic traces have the added advantage of being controllable, and hence, can be used to make more

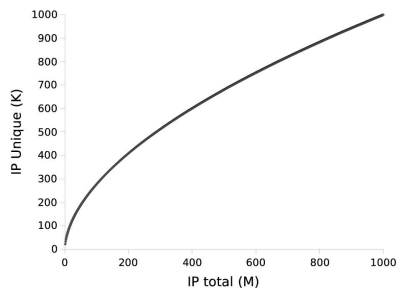

(a) (b)

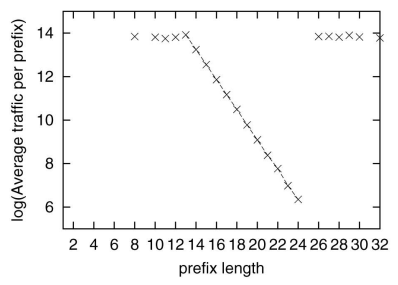

Fig. 3. Characteristics of pld1M trace $(A=10, \theta=1.8)$. (a) Footprint curve. (b) Prefix length distribution.

comprehensive evaluations. Two trace generation methods, randIP and randNET, have been in use [2], [17], [38]. In randIP, a random 32-bit IP-address is generated and included in the trace if it matches an entry in the routing table. In randNET, a random prefix is chosen from the routing table and expanded to 32 bits. Traces generated by these schemes do not match the characteristics of real traces [22], [32]. More specifically:

1. Both randNET and randIP do not follow the prefix length distribution of real traces [22]. It is observed that the log of the average number of addresses that hit a prefix of a given length decreases linearly with increasing prefix length, for prefix lengths ranging from 13 to 24 . In particular, in this range, as the prefix length increases by one, the log of the mean number of hits per prefix decreases by 0.69 . Although the plot for randIP follows a similar behavior, its slope is much higher. On the other hand, in randNET, the average number of hits for a prefix of a given prefix length remains constant as prefix length increases. The authors of [22] also observe that, unlike randNET and randIP, only a small percentage of the traffic (about 5 percent) hits prefixes outside the prefix length range of 13 to 24 bits.

2. Both the randNET and randIP schemes do not preserve locality characteristics of real traces. With a uniform random number generator used in randNET and randIP, it is highly unlikely that an address that is referred in the recent past will be encountered again in the near future. Hence, both the schemes do not conform to the tenets of temporal locality.

Our trace generation procedure plugs in observation 1 into the LRU stack model [32], [34] to produce a methodology which can emulate both the prefix length distribution and the locality characteristics of real traces. The LRU stack algorithm uses two primary parameters, $A$ and $\theta$, which are used to determine the cumulative distribution function (cdf) for the hit index. The cdf is such that smaller hit indexes are preferred over larger ones. A random variate index which follows the cdf of the hit index is generated. If index points to an entry in the stack, that entry is moved to the top of the stack and the stack is readjusted by moving down all entries upto $($ index -1$)$ by one position. If the index is beyond the current size of the stack, all entries are moved down by one position and a new entry is accommodated at the empty top position. The physical significance of $A$ and $\theta$, and the derivation of the cdf of the hit index can be found in [34]. The important point to note is that by increasing the value of $\theta$, the locality in trace can be increased. Fig. 3 shows the footprint curve for one of our synthetically generated traces with $A=10$ and $\theta=1$.8. It can be seen that this curve depicts a trend similar to the footprint curve of a real trace [32]. 


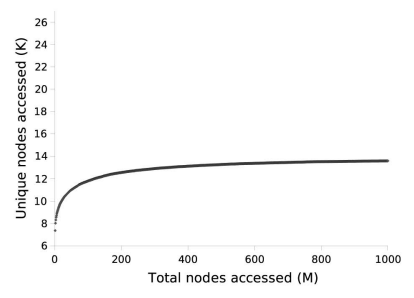

(a)

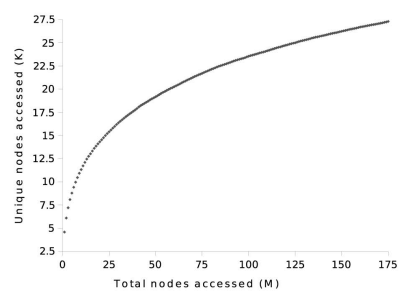

(b)
Fig. 4. Footprint curves. (a) LO nodes. (b) LL nodes.

One question that is not answered by [32] is How to choose a new address to add to the stack? Observation 1 provides us with a natural choice. The new entry should be chosen so that the prefix length distribution of the synthetic trace follows that of realistic traces. We make use of observation 1 to construct the probability mass function for prefix lengths by assigning probabilities for hitting each prefix length. While doing so we ensure that 95 percent of traffic hits prefixes of prefix length 13-24. The remaining 5 percent traffic is uniformly distributed among the prefixes outside this range. All prefixes of a given prefix length are assumed to be equally likely to be picked; hence, once a prefix length is chosen, a prefix of the given prefix length $n$ can be chosen at random. This prefix is then expanded to 32 bits by padding it with a uniformly distributed random number between 0 and $2^{32-n}$.

In Fig. 3b, we plot the log of average traffic per prefix against the prefix length for one of our synthetically generated trace (pld1M). The slope of the line for prefix lengths in the range 13-24 matches well with the real trace used in [22]. It is to be noted that though the average traffic per prefix outside the range 13-24 is high, there are a very few prefixes outside this range, and these prefixes contribute to only 5 percent of the traffic.

\section{HSCA AND Weight-Based RePLACEMENT}

In this section, we motivate the choice of a Heterogeneously Segmented Cache Architecture and describe its operation. We also propose a weight-based replacement policy to enhance the performance of HSCA.

\subsection{Why HSCA?}

The unified cache scheme of [2] does not take into consideration the locality characteristics of the nodes of trie. Each lookup always incurs a LO node access, but may not necessarily visit lower levels. We observe that in realistic traces, only 17 percent of the lookups visit LL nodes. In addition, less than 25 percent of LO nodes are useful (nonsuperfluous) nodes. This implies that a large number of accesses go to a small set of nodes. A plot of the number of unique nodes visited in an LC-trie (footprint curve) for an address trace of one billion packets (refer Fig. 4) reflects this observation. These findings lead us to the choice of a partitioned cache memory with two segments: one cache explicitly for storing the LO nodes and the other cache for storing LL nodes. As only 17 percent of accesses that are incurred by LO cache are incurred by the LL cache, it is natural to have a much smaller LL cache. Based on the above data, we assume an LO size to LL size ratio of 8:1. Such a segmented organization has various advantages. First, it ensures that locality among accesses within each segment is more or less homogeneous. Second, the segmentation prevents the pollution of the LO nodes cache with low

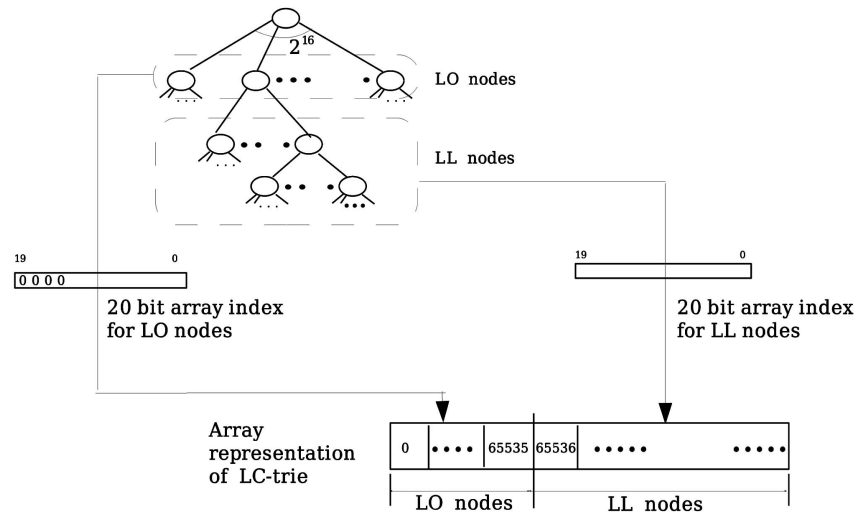

Fig. 5. LC-trie and its array representation.

locality LL nodes which would just waste LO node cache space. Third, it allows each segmentation cache to be optimized separately.

\subsection{Working of HSCA}

An LC-trie is laid out in an array (refer to Fig. 5) with nodes closest to the root (LO nodes) preceding the remaining nodes (LL nodes) in the array. Once the trie is laid out in this way, the index of the array determines the memory address in the memory space (the size of the nodes is a power of 2). Assuming a root branching factor of $2^{16}$ as suggested in [2], [23], the LO nodes will have array index values in the range $0-65,535$, while the LL nodes will have indexes greater than 65,535 . We assume a 20 bit representation for the array index that can accommodate an LC-trie of $2^{20}$ nodes. This many nodes is sufficient for contemporary routing tables [2], [23]. To form a $k$-bit index for either LO cache or LL cache, we use the least significant $k$ bits (bits 0 to $k-1$ ) of the array index. As only bits 0 to 15 can vary for LO nodes, it is sufficient to tag bits $k$ to 16 for the LO cache. However, for the LL cache, $20-k$ most significant bits form the tag. In all our experiments we keep the number of lines in LL cache to be $1 / 8$ th of the number of lines of the LO cache. Hence, if we use 12 bits to index $\mathrm{LO}$ cache, we need to use only 9 bits to index the LL cache. As the node accesses do not exhibit any spatial locality [6], [2], we use the size of a single node as the cache block size. Though we evaluate the HSCA for an LC-trie data structure, it is to be noted that as most trie-based schemes use a large root branching factor [29], [4], the same cache organization can be used for other trie-based lookup algorithms as well.

\subsection{Weight-Based Replacement Policy}

One advantage of using separate caches for LO and LL nodes is that it allows each segment to be optimized individually. As the LO nodes are accessed more frequently and a large amount of cache space is dedicated to the LO nodes cache, it is natural to focus optimization on this cache segment. Below we propose a improved replacement policy for the LO cache. Most replacement policies can be thought of as replacing the cache line with the least weight. In LRU, the weight is based on the recency of use, with most recently used line being given the maximum weight. In FIFO, the weight is the reciprocal of the lifetime in cache, and in LFU, it is the number of accesses. At core routers, where locality is expected to be less, these replacement schemes can be improved by finding better weight assignment schemes. We propose a weight-based replacement policy for the 
TABLE 1

Traces Used for Performance Evaluation

\begin{tabular}{||l|l|l|l|l||}
\hline Name & $A$ & $\theta$ & \multicolumn{2}{|c||}{ Total addresses } \\
\cline { 4 - 5 } & & & FUNET router & Oregon router \\
\hline pld1M & 10 & 1.8 & $1 \mathrm{~B}$ & $1 \mathrm{~B}$ \\
pld10M & 10 & 1.5 & $1 \mathrm{~B}$ & $1 \mathrm{~B}$ \\
pld100M & 10 & 1.2857 & $275 \mathrm{M}$ & $150 \mathrm{M}$ \\
pld1B & - & - & $1 \mathrm{~B}$ & $1 \mathrm{~B}$ \\
\hline
\end{tabular}

LO cache that statically assigns a weight to each of the LO nodes. These weights are based on two observations:

1. A majority of the traffic from real traces hits prefixes whose length ranges from 13 to 24 . Within this range, as prefix length increases, the average number of hits for a prefix of a given prefix length decreases [22].

2. With a branching factor of $2^{16}$ at root, prefixes of length $p<16$ would have $2^{16-p}$ LO nodes pointing to the same forwarding table entry. Therefore, an LO node representing a prefix of length $p$ is only covering $1 / 2^{16-p}$ prefixes.

Based on the above, we propose the following weight assignment

- $\quad$ LO nodes corresponding to prefixes of length 8-12 are given weights from 0 to 4 , respectively.

- LO nodes corresponding to prefixes of length 13-16 are given a weight of 7 .

- The remaining LO nodes are sorted in ascending order of the number of prefixes the subtrie rooted at that node covers. The lower half of this sorted set is given a weight 5 and the upper half is given a weight of 6 .

As the weights are from 0 to 7 , we need 3 bits to represent them. This field can be added to the trie node structure. To make a replacement decision, the weights of the nodes are read and the lowest weight node is chosen for replacement. In case of a tie, LRU replacement is used.

\section{Performance Evaluation}

To evaluate the performance of HSCA and the optimizations proposed in Section 5, we use a trace-driven simulation methodology. To measure the misses incurred, we use the dineroIV cache simulator with some modifications. The modifications are made to accommodate our weight-based replacement policy and simulate for caches of odd sizes (number of sets is not a power of two). For the odd-sized caches, indexing is done using the remainder obtained by dividing the address (trie index) by the number of sets.

We use two routing tables for evaluation, the FUNET routing table used in literature [23] and a recent routing table from the Oregon core router made available through the Route Views Project [41]. The FUNET table contains 41,578 entries and its LC-trie representation leads to 128,865 trie nodes. While the Oregon router contains 161,516 entries and 338,193 LC-trie nodes. The traces used for simulation are generated using the attribute preserving trace generation methodology described previously (Section 4).

The traces used for performance comparison along with the parameters used to generate them and the length of the trace are listed in Table 1. Traces pld1M, pld10M, and pld100M were generated using the methodology. Trace pld1B was generated without using the LRU stack, but conforming to prefix length distribution characteristic of realistic traces. The suffix in the trace name gives us a measure of the number of unique entires that would be in the trace if it were to contain a total of 1 billion entries. The total number of addresses in each trace is $1 \mathrm{~B}$ (with the exception of pld100M), which is two- to three-order magnitudes higher than used in earlier studies [21], [22], [2]. Traces pld1M and pld10M which have more locality represent traffic seen by edge routers. While pld100M and pld1B, which have lower locality, are representative of traffic seen by core routers [25]. Note that as the trace pld1B contains no induced locality, it can be thought of as a worst-case traffic scenario. HSCA could still help in this scenario as even though there is no locality in the trace, there could still be locality in accesses to the trie used for forwarding.

Throughout this section, we will refer to the size of HSCA in terms of the number of lines in the LO cache. Note that given the number of LO cache lines, we can determine the number of LL cache lines. For example, an 8K HSCA refers to an HSCA with $8 \mathrm{~K}$ LO cache lines and 1K LL cache lines. We evaluate the performance of $2 \mathrm{~K}, 4 \mathrm{~K}$, and $8 \mathrm{~K}$ HSCA with associativities 1, 2, 4, and 8, respectively. Comparisons with existing cache schemes are done both in terms of reduction in miss rate and reduction in average memory access time. The miss rates are compared by normalizing them against the base unified/IHARC miss rate. This is done to highlight the performance difference. The memory access speedup metric takes into consideration the effects of both the absolute miss rate and the percentage reduction in number of misses. The speedup in memory access time is measured as [14]

$$
\text { speedup_lower_bound }=\frac{h t+m r_{\text {base }} * m p}{h t+m r_{H S C A} * m p},
$$

where $m r_{\text {base }}$ is the miss rate of the base cache (unified/ IHARC) and $m r_{H S C A}$ is the miss rate of HSCA (both segments included). We assume a hit time $(h t)$ of 1 clock cycle and a miss penalty $(m p)$ of 100 clock cycles [2], [21]. As the information in the auxiliary tables can be stored internally within the LC-trie itself [27], the only memory accesses are trie node accesses.

\subsection{Performance Comparison with the Unified Cache}

We compare the performance of an HSCA of a given size with a unified cache having the same number of total lines $(2 \mathrm{~K}, 4 \mathrm{~K}$, and $8 \mathrm{~K}$ HSCA would correspond to a $2.25 \mathrm{~K}, 4.5 \mathrm{~K}$, and $9 \mathrm{~K}$ entry unified caches, respectively) and same associativity. Figs. 6 and 7 compare the miss rate of HSCA with unified, while Fig. 8 plots the speedup in average memory access time. We only plot the performance for two representative pairings of routing table and trace to save space. For all cache sizes and associativities HSCA performs better. A performance improvement of up to 25 percent in miss rate and a 32 percent memory access speedup is observed. The difference in performance can be attributed to the segmented organization of HSCA. By ensuring that only nodes with similar locality share a segment, HSCA prevents pollution of cache space. The unified cache, on the other hand, incurs additional misses as nodes with heterogeneous characteristics compete for the same space. Further, it can be seen that the performance improvement of HSCA gets amplified further with increase in cache size. This again implies that HSCA makes better use of the available cache lines than the unified cache. 


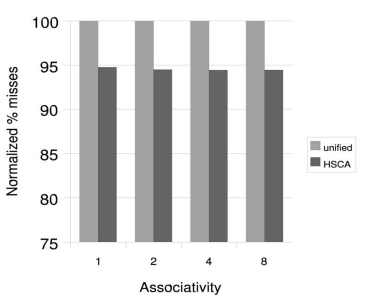

(a)

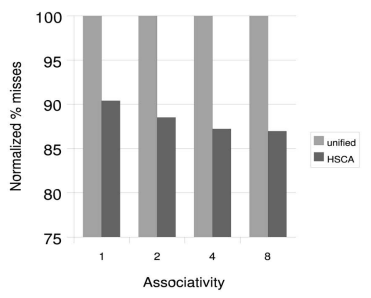

(b)

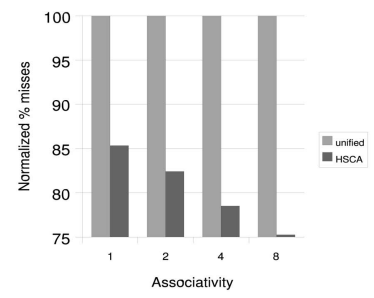

(c)

Fig. 6. Miss rate comparison, Oregon, pld1B. (a) 2K HSCA. (b) 4K HSCA. (c) 8K HSCA.

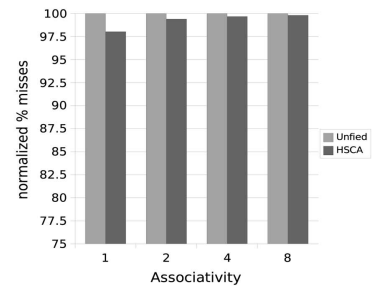

(a)

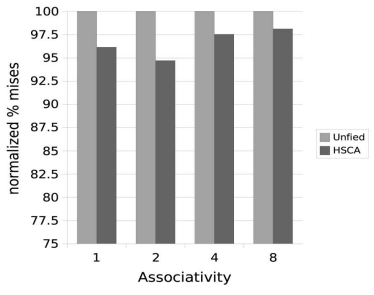

(b)

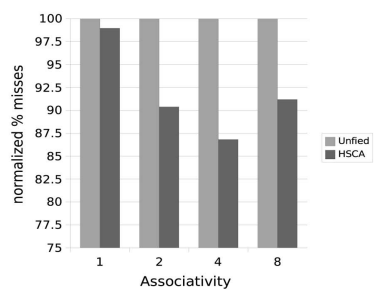

(c)

Fig. 7. Miss rate comparison, FUNET, pld10M. (a) 2K HSCA. (b) 4K HSCA. (c) 8K HSCA.

\subsection{Impact of Locality on Performance Improvement}

Fig. 9 shows the impact of locality in trace on the performance of HSCA. The plot shows that there is an increase in the relative performance of HSCA as locality reduces (especially for higher cache sizes). In fact, the best miss improvement is observed for an 8K HSCA with a pld1B trace (lowest locality). For this configuration, there is a 17.5 percent reduction in number of misses for the FUNET table and 25 percent for the Oregon router. The corresponding speedups in memory access time are 19.9 percent and 32.1 percent, respectively. This indicates that by segregating the nodes with high locality from the low locality nodes, HSCA is successful in extracting performance even in worst-case traffic. This happens as by preventing pollution of the LO cache, HSCA extracts locality among LO nodes even when the traffic does not contain locality.

\subsection{Performance Comparison with IHARC}

The comparison between HSCA and the unified cache is simple as both cache the nodes of an LC-trie. However, the comparison of HSCA with IHARC is not as straightforward. Below we describe the complications that arise and provide a work around.

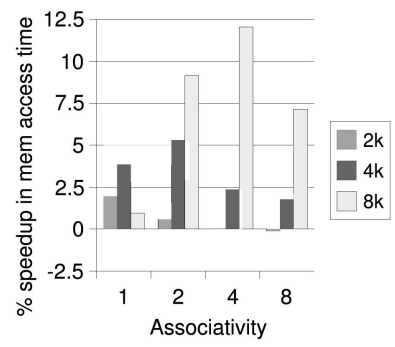

(a)

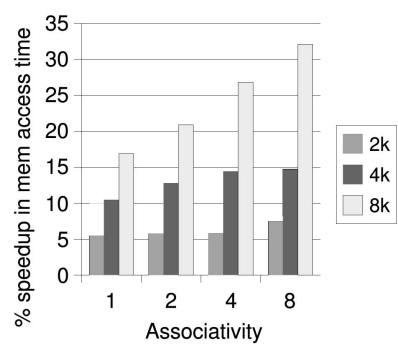

(b)
Fig. 8. Speedup in memory access time over unified. (a) FUNET, pld10M. (b) Oregon, pld1B.
1. Counting misses: As HSCA caches nodes of a trie and IHARC caches lookup results, the misses of the two cannot be compared directly. A single miss from IHARC can lead to many memory accesses as multiple trie nodes may have to be accessed. To overcome this, we measure IHARC misses in terms of the number of LC-trie accesses incurred by the IP-addresses that miss the IHARC result cache.

2. Node structure and size: To avoid maintaining auxiliary tables the modified LC-trie adds two fields port and string to the original LC trie (refer to Fig. 10). The field Port stores the result of the lookup, while string is needed to verify if the address indeed matches the prefix of the node, as some of the address bits could have been skipped.

We can further cut down on the size of the nodes by utilizing the properties of an LC-trie. The string field needs to be stored only if there has been a path compression along the path. As from root the first 16 bits are all used to reach an LO node, there is no need to store the string for LO nodes. Also, the pointer field only points to the leftmost child at level two, 19 bits are sufficient for the pointer field of the LO nodes. For the same reason, even for the LL nodes, it is sufficient to store only the lower 16 bits. This leads us to the node structure of Fig. 11.

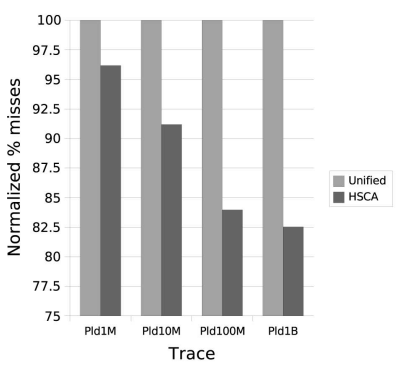

(a)

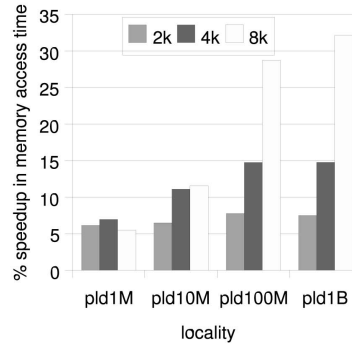

(b)
Fig. 9. Impact of locality in trace. (a) Impact on miss rate, 8K eight-way HSCA, FUNET. (b) Impact on average memory access time, Oregon. 


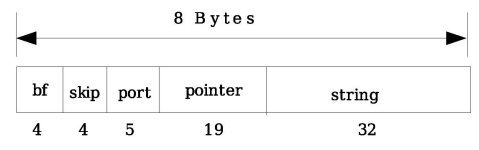

Fig. 10. Node structure for modified LC-trie.

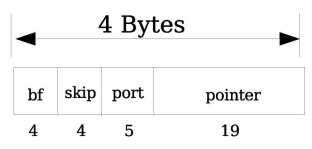

(a)

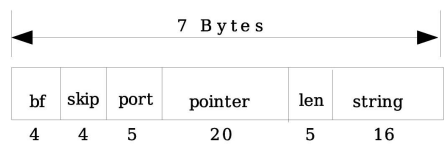

(b)
Fig. 11. Node structure for HSCA. (a) LO node. (b) LL node.

TABLE 2

Total Sizes of HSCA and IHARC (in bytes)

\begin{tabular}{||l|l|l|l|}
\hline number of sets & IHARCsize(B) & HSCAsize(B) & Size Ratio \\
\hline 256 & 1696 & 1692 & 99.7 \\
512 & 3264 & 3312 & 101.5 \\
1024 & 6272 & 6480 & 103.3 \\
2048 & 12032 & 12672 & 105.3 \\
4096 & 23040 & 24768 & 107.5 \\
8192 & 44032 & 48384 & 109.9 \\
\hline
\end{tabular}

The length field (len) is used to identify how many of the bits of the address should match the string field. This field is added to save on the need to remember how many bits of the address have been utilized. Because of the different sizes of the nodes, we would now need to use two arrays to store the trie: one for LO nodes and one for the LL nodes.

3. Making a fair comparison between IHARC and HSCA: A fair comparison can only be made if the two caches are of identical sizes. The IHARC scheme requires a large tag space as it stores a mask in addition to the conventional tag. The tag and the mask field together require $2 *(32-k)$ bits per line for a cache with $2^{k}$ sets. In comparison, HSCA only needs $(16-k)$ bits for each LO cache line and $(20-j)$ bits for each LL cache line $\left(j=k-3\right.$, as LL has $1 / 8^{\text {th }}$ the sets). On the other hand, each data element of IHARC only needs to store a 5-bit output port field, whereas each entry in HSCA is a node of comparatively larger width (refer Fig. 11). Based on the above, we calculate the actual size (including both tag and data space) needed for each cache configuration (refer to Table 2). We find that it is reasonable to compare an IHARC scheme with $2^{k}$ cache sets with an HSCA with $2^{k}$ sets in LO cache and $2^{k-3}$ sets in the LL cache, as even in the worst case, the HSCA size is within 10 percent of the IHARC size.

\subsection{Performance Results}

In Fig. 12, we compare the misses incurred by HSCA with those of IHARC, and in Fig. 13a, we compare the speedup. To save space, we present results only for the FUNET routing table. HSCA performs 45 percent to 90 percent better for the pld10M trace. Again, as the size increases, we find that the relative performance of HSCA gets better and brings down misses to $1 / 10$ th at $8 \mathrm{~K}$. It is to be noted that this reduction in miss rate results in a few orders of magnitude speedup (almost $8 \times$ ) in memory access time. This large difference in performance can be attributed to the ability of HSCA to exploit the additional locality among accesses to individual trie nodes. IHARC, on the other hand, can only exploit the locality among destination IP-addresses of incoming packets.

\subsection{Impact of Locality on Performance Improvement}

In Figs. 13b and 13c, we plot the impact of locality in trace on performance for $8 \mathrm{~K}$, eight-way associative cache. The performance curves for other cache sizes and associativities exhibit similar trend. The plot shows that HSCA outperforms IHARC for all traces. It is observed that for traces with higher locality (pld1M and pld10M), HSCA reduces the number of misses by a huge margin 60 percent to 80 percent. Unlike the unified cache, the relative performance of IHARC improves relative to HSCA for lower locality traces. However, even with the worst-case locality trace (pld1B), there is a 100 percent speedup in average memory access time for an $8 \mathrm{~K}$ cache.

In summary, the performance improvements gained by HSCA over both the unified cache and IHARC are robust. Lastly, it is to be noted that even though we do not simulate a full system with support for multithreading, some of the effects of multithreading on cache performance will be captured by our methodology. This is because multithreading would only change the order in which packet IP-addresses are processed due to interleaving of packets from multiple threads. This, in turn, will only affect the locality characteristics of the interleaved trace. As our cache organization is robust enough to gain performance under traffic with varying locality characteristics, we can expect that HSCA will do well even in the presence of multithreading. However, it is to be noted that the effect of multithreading on the interleaving of the accesses to the trie nodes will not be captured by our methodology. For example, with our methodology, the stack distance between two accesses to the root node cannot be less than the minimum path length from root to the closest leaf node, while with multithreading, a lower stack distance is observable. A more detailed analysis will be required to understand the complete impact of multithreading on the performance of a trie nodes cache. A full system simulation will also be required to understand the extent to which

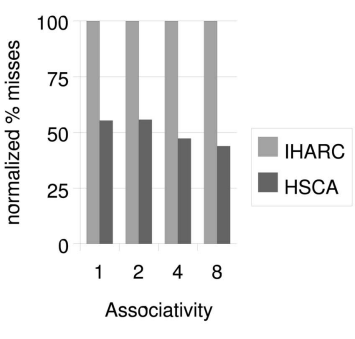

(a)

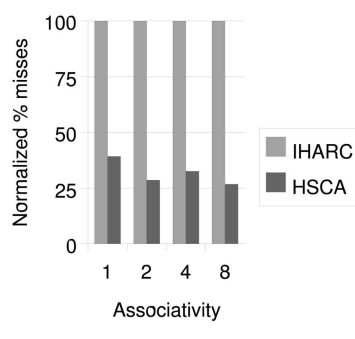

(b)

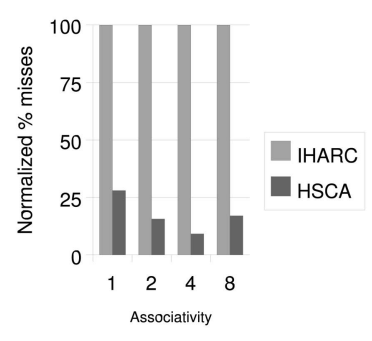

(c)

Fig. 12. Comparison of HSCA with IHARC, pld10M, and FUNET. (a) 2K HSCA. (b) 4K HSCA. (c) 8K HSCA. 


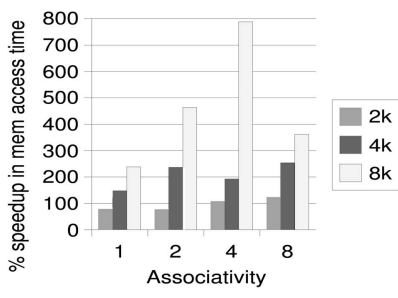

(a)

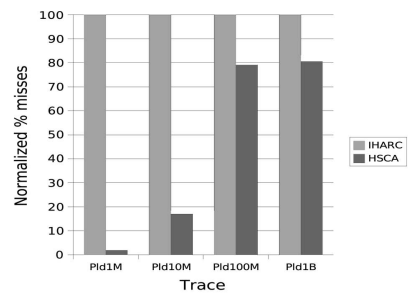

(b)

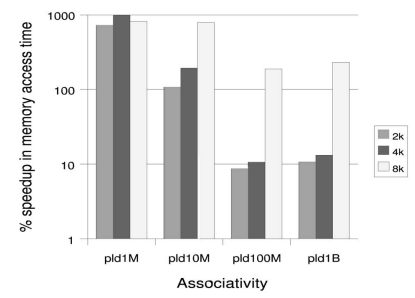

(c)

Fig. 13. HSCA versus IHARC. (a) Speedup in memory access time over IHARC, FUNET, pld10M. (b) Impact of locality on miss rate, 8K HSCA. (c) Impact of locality on memory access time.

multithreading and/or caching will ease the memory bottleneck. Such a study is beyond the scope of this work and is addressed elsewhere [2], [21], [30].

\subsection{Weight-Based Replacement}

Fig. 14 shows the percentage misses of the weight-based replacement policy relative to LRU for the pld100M trace. The corresponding speedup plots are in Fig. 15a. It can be seen that for large cache sizes ( $4 \mathrm{~K}$ and $8 \mathrm{~K}$ ) weight-based replacement outperforms LRU by up to 20 percent in terms of misses and 37 percent in terms of access speedup. This corresponds to an overall memory access time speedup of about 40 percent for an HSCA with weight-based replacement for LO over the unified cache for a pld1B trace. As size or associativity increases, the relative performance improvement gets better. A similar trend is seen for all traces having lesser locality than pld100M (refer to Fig. 15b). For traces having more locality (pld1M and pld10M), LRU replacement performs better. This implies that for core routers, we should use a weight-based replacement for the $\mathrm{LO}$ cache of the HSCA, while at the edge we should use LRU.

\section{Enhancing Performance with Two-Level MAPPING}

The heavy skew of accesses toward LO nodes motivates the need to exploit the $\mathrm{LO}$ cache organization in more detail. One potential source for performance gains is the presence of a large number of superfluous LO nodes interspersed with useful LO nodes. For the routing tables we use, a branching factor of $2^{16}$ results in as many as 75 percent superfluous LO nodes. We find that using a conventional placement function (indexing with lower order bits) for the $\mathrm{LO}$ cache may not uniformly distribute the useful LO nodes among the sets. We now illustrate the drawbacks of conventional one-level mapping-based cache indexing with the help of an example. Then the arguments in favor of two-level

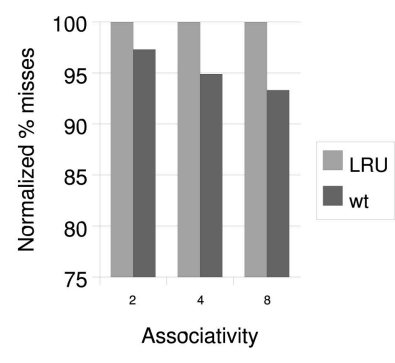

(a)

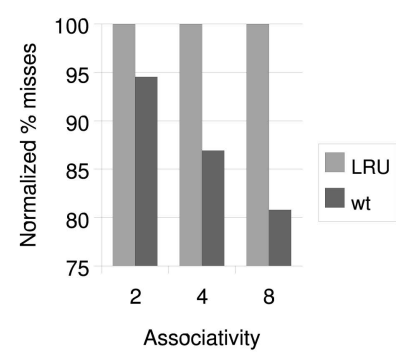

(b)
Fig. 14. Weight-based Replacement versus LRU, HSCA LO. (a) 4K LO. (b) $8 \mathrm{~K}$ LO. mapping are put forth and the motivation for the proposed remapping schemes is provided.

Consider the routing table in Table 3 . The table has 16 entries with a maximum prefix length of 7 . There are four prefixes of length 5, four of length 6 and eight of length 7 . Now, if we consider a root branching factor of $2^{7}$, prefixes of length 5 will be expanded to four nodes each (refer to Table 3), prefixes of length 6 to two nodes each, while each prefix of length 7 will form a single node. These are the only useful LO nodes in the trie. Hence, out of a total of $128 \mathrm{LO}$ nodes, there are 32 (25 percent) useful nodes and the remaining 96 (75 percent) nodes are superfluous. If these useful nodes were to be divided into eight sets, we would ideally want each set to have exactly four nodes.

\subsection{One-Level Mapping-Based Placement}

\subsubsection{Conventional Mapping}

The simplest way to map the nodes into cache sets would be to partition the useful nodes based on the lower order bits. Unfortunately, this naive partitioning will result in an uneven distribution of useful nodes among sets. For the example in Table 3, a 3-bit index would form eight sets with $5,6,4,2,4,3,2$, and 6 nodes, respectively, as shown in Table 4 . Clearly, the mapping is not ideally suited for caching. The highly populated sets would have more conflicts, while the sparsely populated ones will be underutilized.

\subsubsection{Node to Set Mapping with Bit Selection (BS)}

One simple way of improving node to set distribution would be to pick the index bits more intelligently. Instead of using the lower order bits, we can choose the index bits that partition the nodes most uniformly. In order to keep the computational complexity low, we pick the bits one at a time, picking the next bit based on how well it, along with previously chosen bits, partitions the nodes. For the example under consideration, the 3 bits chosen would be

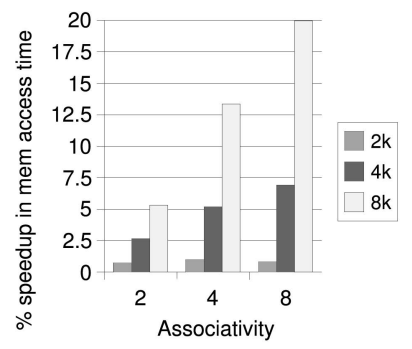

(a)

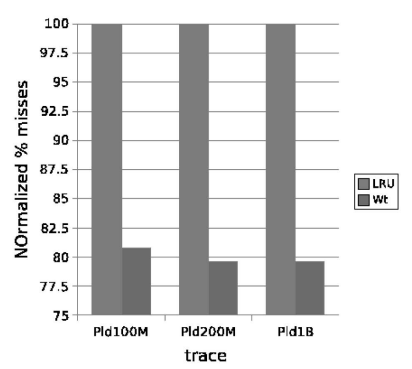

(b)
Fig. 15. Weight-based replacement. (a) Speedup in memory access time over HSCA, FUNET, pld100M. (b) Impact of locality on weightbased replacement. 
TABLE 3

Routing Table

\begin{tabular}{||c|l|c|c|c||}
\hline Sno & prefix & prelen & next-hop & LO nodes \\
\hline 1 & 0001111 & 7 & 3 & 1 \\
\hline 2 & 010000 & 6 & 3 & 2 \\
\hline 3 & 0100010 & 7 & 2 & 1 \\
\hline 4 & 01001 & 5 & 1 & 4 \\
\hline 5 & 0110001 & 7 & 4 & 1 \\
\hline 6 & 0111111 & 7 & 2 & 1 \\
\hline 7 & 100000 & 6 & 1 & 2 \\
\hline 8 & 10001 & 5 & 1 & 4 \\
\hline 9 & 100100 & 6 & 2 & 2 \\
\hline 10 & 1010010 & 7 & 5 & 1 \\
\hline 11 & 110110 & 6 & 3 & 2 \\
\hline 12 & 1101111 & 7 & 4 & 1 \\
\hline 13 & 11100 & 5 & 1 & 4 \\
\hline 14 & 1110100 & 7 & 2 & 1 \\
\hline 15 & 1110111 & 7 & 4 & 1 \\
\hline 16 & 11110 & 5 & 2 & 4 \\
\hline
\end{tabular}

$b_{0}, b_{1}$, and $b_{4}$. When these 3 bits are used, the resulting number of nodes per set is shown in Table 5 . It can be observed that we still have two IPs (sets) with six nodes which are likely to end up having more conflicts than the four IPs (sets) with three nodes each.

The reason for the nonuniform distribution in the above two schemes is that they are very restrictive in cache index selection, i.e., both use a fixed set of index bits to directly determine the mapping from nodes to cache sets. Further, conventional placement does not exploit the knowledge of the distribution of useful LO nodes. It simply uses a predefined function of the address bits to determine the cache index. Even an XOR-based placement scheme uses a predetermined function to form the index and makes no further attempt to achieve a more uniform distribution.

A better mapping scheme would be one that can exploit the knowledge of the distribution of useful nodes and yet be flexible enough to adapt to changes in the routing table. In addition, the flexibility achieved in placement should neither significantly increase the hardware complexity nor increase the hit latency. We achieve these goals through a two-level mapping framework that adds a few decision bits per IP to decide the RP to which it maps. The remapping from IP to RP ensures better distribution; the usage of different decision bits for each IP makes the remapping adaptive and the placement flexible; and as the index is identified by using a few additional multiplexers (as we will see in Section 8), the hit time can still be accommodated in one clock cycle.

\subsection{Two-Level Mapping-Based Placement}

\subsubsection{FLEX}

In FLEX, we use two conventional bits $\left(b_{0}, b_{1}\right)$ to first identify the IPs. In Table 6 , the last two rows show the number of nodes mapping to each set when each of $b_{2}$, $b_{3}, \ldots, b_{6}$ are used in conjunction with $b_{1}$ and $b_{0}$. It can be seen that using the same final bit for all IPs is not ideal. Instead, in FLEX, one among $\left\{b_{2}, b_{3}, b_{4}, b_{5}\right\}$ is chosen to reorganize each IP into two RPs. Note that this bit is chosen on a per IP basis. The chosen bit and number of nodes for each RP are shown in boldface in the last two rows of Table 6. This mapping is better than that achieved by conventional mapping or BS.

\subsubsection{IMAP}

Even though the mapping achieved by FLEX is ideal for $I P_{0}$, $I P_{2}$, and $I P_{3}$, it can be seen from Table 6 that for $I P_{1}$, we are unable to find a bit which would split the IP into two RPs with equal number of nodes. A closer look at $I P_{1}$ (refer Fig. 16) reveals that if we further partition it based on bits $b_{2} b_{3}$ and combine the partition $b_{2} b_{3}=00$ with the partition $b_{2} b_{3}=11$ to form an RP, we get an uniform mapping for $I P_{1}$. This is what we achieve with IMAP. The four partitions of Table 6 are further partitioned based on bits $b_{2}$ and $b_{3}$ to get 16 partitions (refer to Table 7a). Now, in each column, the IP with the most nodes in it is combined with the IP having the least nodes in it (both shown in bold) to form one RP, and the remaining IPs in the column form the other RP. Note that $I P_{2}$ which relies on bit $b_{4}$ to get uniformly remapped in FLEX can still be uniformly remapped in IMAP using $b_{2} \oplus b_{3}$. For the example in consideration, using IMAP, we obtain eight RPs with 5, 5, $3,4,4,4,3$, and 4 nodes in them. In Table 8 , we compare the node to set (IP for one-level mapping and RP for two-level mapping) distribution obtained by the various schemes for the routing table in consideration. It can be observed that the IMAP and FLEX schemes achieve a more uniform distribution of nodes than the base mapping and BS. In Section 9, we quantitatively compare the distributions achieved by the various schemes on real routers.

\subsubsection{V-ASSOC}

Through V-ASSOC, we propose a two-level mapping-based variable associativity cache that chooses a cache set with a higher (lower) associativity for RPs with more (fewer) nodes. More specifically, the larger two IPs per column of Table $7 \mathrm{~b}$ (shown in bold) are mapped to larger RPs corresponding to cache sets with more number of lines (higher associativity) in them, while the remaining two are mapped to smaller RPs corresponding to sets with lower associativity. This scheme attempts to distribute the percentage of each partition that can be cached more uniformly.

\section{Cache Organization}

In this section, we describe the cache organization needed to implement each of the two-level mapping-based schemes. We also propose modifications to the cache organization to elegantly fit in two-level mapping by keeping the increase in

TABLE 4

Initial Partitions Formed using bits $b_{1}, b_{2}$ and $b_{3}$

\begin{tabular}{|c|c|c|c|c|c|c|c|}
\hline$I P_{0}$ & $I P_{1}$ & $I P_{2}$ & $I P_{3}$ & $I P_{4}$ & $I P_{5}$ & $I P_{6}$ & $I P_{7}$ \\
\hline $\begin{array}{llllllllllll} & 1 & 0 & 0 & 0 & 0\end{array}$ & $0 \begin{array}{llllll}0 & 1 & 0 & 0 & 0 & 0\end{array}$ & 11110010 & 11110011 & 010100100 & 010100101 & $\begin{array}{llllllllll}0 & 1 & 0 & 1 & 1\end{array}$ & 0100111 \\
\hline $\begin{array}{llllllll}1 & 0 & 0 & 0 & 0 & 0 & 0\end{array}$ & $\begin{array}{llllll}0 & 1 & 0 & 0 & 0 & 0\end{array}$ & $\begin{array}{llllll}0 & 1 & 1 & 0 & 0 & 1\end{array}$ & 1111011 & $\begin{array}{llllllllllll}1 & 0 & 1 & 0 & 0\end{array}$ & $\begin{array}{lllll}1 & 0 & 0 & 1 & 0\end{array}$ & 10000110 & 1000111 \\
\hline 111100000 & $\begin{array}{lllllll}1 & 0 & 0 & 0 & 0 & 0 & 1\end{array}$ & $\begin{array}{lllllll}1 & 0 & 1 & 0 & 0 & 1 & 0\end{array}$ & - & 1110100 & 1101101 & - & 1110111 \\
\hline $\begin{array}{lllllll}1 & 0 & 1 & 0 & 0 & 0\end{array}$ & 11100001 & 1111010 & - & 1101100 & - & - & 0001111 \\
\hline 1111000 & 1001001 & - & - & - & - & - & 0111111 \\
\hline & 1111001 & - & - & - & - & - & 1101111 \\
\hline 5 & 6 & 4 & 2 & 4 & 3 & 2 & 6 \\
\hline
\end{tabular}


TABLE 5

Number of Nodes per Partition for Eight IPs Formed Using Bits $b_{0}, b_{1}$, and $b_{4}$, Chosen by BS

\begin{tabular}{|c|c|c|c|c|c|}
\hline & \multicolumn{4}{|c|}{$b_{1} b_{0}$} \\
\hline & & 00 & 01 & 10 & 11 \\
\hline \multirow{2}{*}{$b_{4}$} & 0 & 6 & 6 & 3 & 4 \\
\hline & 1 & 3 & 3 & 3 & 4 \\
\hline
\end{tabular}

TABLE 6

Initial Partitions Formed Using Bits $b_{0}, b_{1}$

\begin{tabular}{|c|c|c|c|}
\hline$I P_{0}$ & $I P_{1}$ & $I P_{2}$ & $I P_{3}$ \\
\hline$b_{6} b_{5} \ldots \ldots . b_{1} b_{0}$ & $b_{6} b_{5} \ldots \ldots . b_{1} b_{0}$ & $b_{6} b_{5} \ldots \ldots . . . b_{1} b_{0}$ & $b_{6} b_{5} \ldots \ldots . . . b_{1} b_{0}$ \\
\hline $0 \begin{array}{lllllllll}0 & 0 & 0 & 0 & 0 & 0 & 0\end{array}$ & $0 \begin{array}{lllllll}0 & 1 & 0 & 0 & 0 & 0 & 1\end{array}$ & 11110010 & 11110011 \\
\hline 1000000 & 0110001 & 0100010 & 1111011 \\
\hline 11100000 & 10000001 & 1010010 & 0100111 \\
\hline 10001000 & 111100001 & 1111010 & 1000111 \\
\hline 11111000 & 10001001 & 01000110 & 1110111 \\
\hline 1101100 & 1111001 & 10000110 & 0001111 \\
\hline 0100100 & 0100101 & - & 0111111 \\
\hline 1000100 & 1000101 & - & 1101111 \\
\hline 1110100 & 1101101 & - & - \\
\hline 23665 & 33666 & 22354 & 32442 \\
\hline 76334 & 66333 & 44312 & 56446 \\
\hline
\end{tabular}

\begin{tabular}{|c|c|c|}
\hline$b_{4} b_{3}$ & & $b_{4} b_{3}$ \\
\hline $\begin{array}{lllllllllllllll}0 & 1 & 0 & 0 & 0\end{array}$ & & $\begin{array}{llll:llllllllll}0 & 1 & 0 & 0 & 0 & 1\end{array}$ \\
\hline $\begin{array}{ll:lllllllllll}0 & 1 & 0 & 0 & 0 & 1\end{array}$ & & $\begin{array}{lllllllllllllllllll}0 & 1 & 0 & 0\end{array}$ \\
\hline 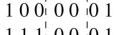 & \multirow{5}{*}{ most nodes } & 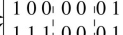 \\
\hline $\begin{array}{llllllllll}0 & 1 & 0 & 0 & 1 & 0\end{array}$ & & 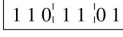 \\
\hline $\begin{array}{ll:lllll}100 & 01 & 1 \\
\end{array}$ & & $\begin{array}{lllllllll}0 & 1 & 0 & 0 & 0\end{array}$ \\
\hline $\begin{array}{llllllllllll}1 & 0 & 0 & 1 \\
1\end{array}$ & & $\begin{array}{lllll}1 & 0 & 0 & 0 & 1\end{array}$ \\
\hline 1 $110: 11: 101$ & & 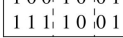 \\
\hline
\end{tabular}

Fig. 16. Remapping of $I P_{1}$ to RPs using IMAP.

access time to a bare minimum. Section 9.5 provides a detailed timing analysis based on HSPICE simulation. To help understand these modifications, some background on the physical organization of a conventional cache is required. Below we provide a brief background on this with an emphasis on the components that contribute to the placement function.

\subsection{Basic Cache Organization}

The access time of a cache is not only dependent on the highlevel cache parameters (cache size, block size associativity, etc.), but also on the physical organization of the tag and data arrays. Typically, both the tag and data arrays of a cache are organized into multiple subarrays. The exact choice of the number and size of the subarrays is determined by minimizing a weighted function of cache access time, power consumption, and area estimates [33]. Fig. 17 shows the physical organization for an 32-KB two-way associative cache with 4B cache lines; this corresponds to $8 \mathrm{~K}$ line twoway associative LO node cache. This cache will have 4,096 sets with $8 \mathrm{~B}$ of data per set. For the example considered (refer to Fig. 17), the data array has four subarrays (DS0-DS3) and each subarray contains one-fourth of the sets $(4,096 / 4=1,024)$. The sets within each subarray are arranged so that four sets (256 bits) are along a single row and there are 256 such rows. The optimal number of subarrays and number of sets per row was obtained using the Cacti tool [33]. On a cache reference, one row in each subarray is simultaneously read and passed on to the column multiplexers. The final data set is chosen from among 16 sets through a 4 to 16 column decoder. The tag array has two subarrays that are simultaneously accessed on
TABLE 7

Number of Nodes per Partition Using Lower Order Bits (a) 16 IPs. (b) 8 IPs

\begin{tabular}{|c|c|c|c|c|c|}
\hline & \multicolumn{4}{|c|}{$b_{1} b_{0}$} \\
\hline & & 00 & 01 & 10 & 11 \\
\hline \multirow{4}{*}{$b_{3} b_{2}$} & 00 & 3 & 4 & 3 & $\mathbf{1}$ \\
\hline & 01 & 3 & 2 & 2 & 3 \\
\hline & 10 & 2 & 2 & 1 & 1 \\
\hline & 11 & 1 & 1 & 0 & 3 \\
\hline
\end{tabular}

(a)

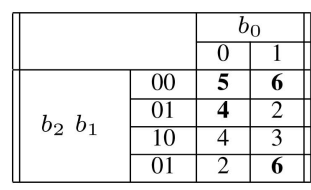

(b)
TABLE 8

Number of Partitions Having the Given Number of Nodes

\begin{tabular}{||c|c|c|c|c|}
\hline \multirow{2}{*}{$\begin{array}{c}\text { Number of Nodes } \\
\text { per set }\end{array}$} & \multicolumn{4}{|c|}{ Number of sets } \\
\cline { 2 - 5 } & base & BS & FLEX & IMAP \\
\hline 1 & 0 & 0 & 0 & 0 \\
\hline 2 & 2 & 0 & 0 & 0 \\
\hline 3 & 1 & 4 & 3 & 2 \\
\hline 4 & 2 & 2 & 3 & 4 \\
\hline 5 & 1 & 0 & 1 & 2 \\
\hline 6 & 2 & 2 & 1 & 0 \\
\hline 7 & 0 & 0 & 0 & 0 \\
\hline
\end{tabular}

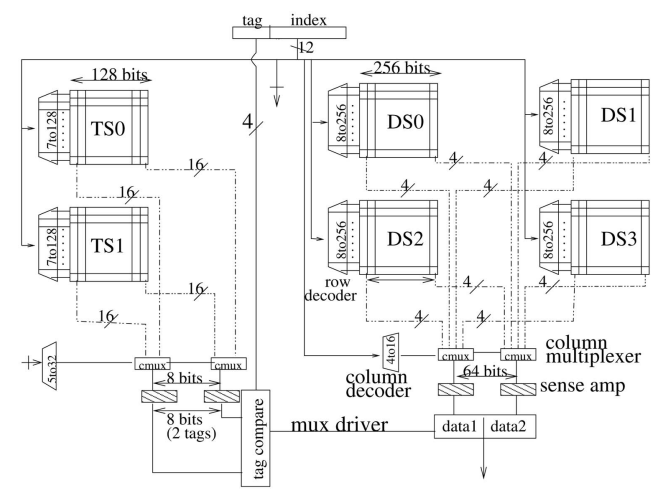

Fig. 17. Multisubarray cache organization.

a reference. Each subarray contains tags corresponding to 16 sets in a row and 128 such rows. With an address of 16 bits, the tag for each cache line is 4 bits wide. Hence, with 16 sets per row of the subarray, each row has 128 bit cells. Again the appropriate set is chosen through a column decoder and multiplexer.

Typically the column decoder is small and fast, whereas the row decoder is slow [39]. The two decodings happen simultaneously and determine the address to set mapping. As the row decoder is slower, it determines the critical path of the placement function. The delay incurred by the row decoder depends not only on the number of bits decoded, but also on the number of bit cells being driven. More details on the cache organization can be found in CACTI's manuals [33], [39].

\subsection{Two-Level Mapping-Based Placement}

All schemes make use of a small number of decision bits to add the required flexibility. The computation of these bits is simple and can be done offline without affecting the performance of the router. The operation of all the schemes primarily involves the identification of two (four for V-ASSOC) candidate sets based on conventional bits, and then, choosing one among them based on the decision bits. As the tag match, miss handling and replacement mechanisms are implemented in a manner identical to a conventional 


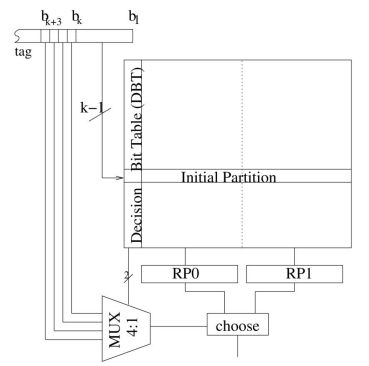

Fig. 18. Cache organization for FLEX.

cache; in the rest of the section, we will focus on the identification of the set to which an address maps (placement). Note that the schemes are applicable to both directmapped and set-associative caches. In order to accommodate the remapping part of two-level mapping into the cache organization, we propose certain modifications to the placement function keeping access time in mind.

\subsection{Cache Architecture for FLEX}

As illustrated in the previous section, FLEX can flexibly choose its final index bit from among four higher order bits, this information is stored using two decision bits per IP. To map a memory address to one among $2^{k}$ sets, traditional placement will use bits $\left\langle b_{k}, \ldots, b_{1}>\right.$ of the line address. In FLEX, $(k-1)$ bits $\left\langle b_{k-1}, \ldots, b_{1}\right\rangle$ are used to map the address to one IP. One among two candidate RPs corresponding to this IP is decided based on one of the next four higher order bits, $\left\langle b_{k+3} \ldots b_{k}\right\rangle$. The logical organization is shown in Fig. 18. The bit used is determined by the decision-bits corresponding to the IP. Note that for each IP, a different final bit can be chosen based on which bit reduces conflicts most.

Physically, the DBT can be organized as shown in Fig. 19. The figure depicts a $8 \mathrm{~K}$ line two-way cache. To minimize wire delays, we propose that the DBT be replicated and placed alongside the data as well as the tag array. The organization is further optimized by placing the DBT alongside the subarray closest to the column multiplexers. In addition, we maintain the number of rows in the DBT to be the same as that in the corresponding (data/tag) subarray. This is because, though the organization of DBT could be optimized by putting more bits along the row, keeping the same number of rows makes it easier to place it (physically) alongside the subarray. Note that the number of columns in the DBT will be much fewer. As mentioned earlier, the row decoder determines the critical path of the placement function. As a consequence, the bits to decode for

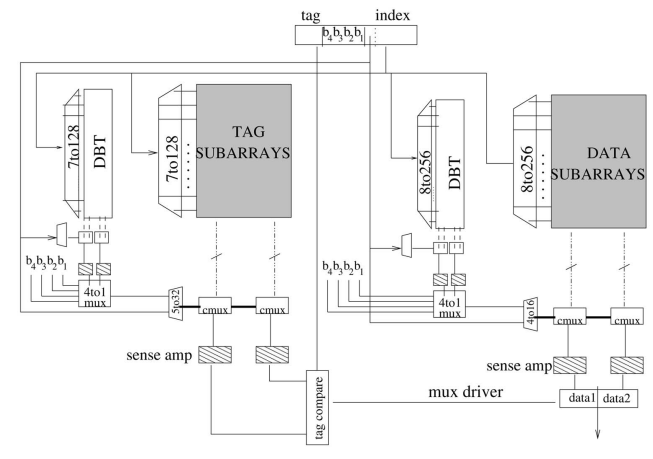

Fig. 19. Physical organization for $8 \mathrm{~K}$ line two-way cache.

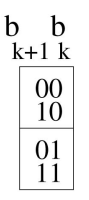

(a)

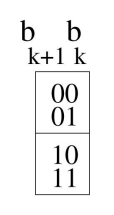

(b)

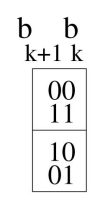

(c)
Fig. 20. Possible combinations of bits $b_{k}$ and $b_{k+1}$ for a set. The bit used to identify the sets is depicted alongside. (a) Case 1: $b_{k}$. (b) Case 2: $b_{k+1}$. (c) Case 3: $b_{k} \oplus b_{k+1}$.

the column decoder can be supplied with a small additional delay without incurring an overall access delay. The row decoders of the data/tag subarrays drive many more bits in comparison to the row decoder of the DBT. Hence, the delay incurred in accessing the DBT (having only few columns), a small 4:1 multiplexer and a fast column decoder (at worst 5 to 32), can be overlapped with the delay incurred by the row decoder of the tag/data subarray. We validate this claim through a detailed timing analysis in Section 9.5.

One final point to note is that whenever the trie used for forwarding is changed and the cache is flushed, the decision bits need to be recomputed. The recomputation takes less than 30 milliseconds real time (measured using the Linux time command) on a $2 \mathrm{GHz}$ Pentium 4 processor. This can easily be done offline without affecting performance.

\subsection{Cache Architecture for IMAP}

As explained previously, IMAP combines the best and worst partition within a group ${ }^{1}$ of four partitions. In IMAP, we start with double the number of IPs $\left(2^{k+1}\right)$ and divide them into groups such that partitions within a group differ only in bits $b_{k}$ and $b_{k+1}$. The number of ways of combining four partitions of a group into two sets (RPs) of two partitions each is given by

$$
N_{I M A P}=\frac{4 !}{2 ! 2 ! 2 !}=3 \text {. }
$$

These three possible combinations of $b_{k}$ and $b_{k+1}$ are depicted in Fig. 20. It can be seen that for Case 1 of Fig. 20, $b_{k}$ identifies the mapping from partitions to sets. Similarly, for Case 2 and Case 3, bit $b_{k}$ and $b_{k} \oplus b_{k+1}$, respectively, will identify the mapping.

For a given address, bits $\left\langle b_{k-1}, \ldots, b_{1}\right\rangle$ are used to index into the appropriate group (four IPs) and pick out the two candidate sets (RPs) (refer to Fig. 22a). For each group, the Case to which it conforms is indicated by the means of the two decision bits. The corresponding final index bit is chosen based on the Case and used to identify the set among the candidates. The physical cache organization for IMAP is identical to that for FLEX. Only the inputs to the 4:1 mux would differ.

\subsection{Cache Architecture for V-ASSOC}

The lower order $k$ bits are used to partition the nodes into $2^{k}$ IPs. As in IMAP, V-ASSOC then divides the partitions into four groups such that the IPs in a group differ only in bits $b_{k-1}$ and $b_{k}$. The larger two IPs of the group are identified as two larger RPs and mapped into sets with higher associativity, and the remaining two partitions are identified as two smaller RPs and mapped to sets with lower

1. The columns of the previous section (Table 7a) are being referred to by a more formal term groups here. 


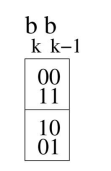

(a)

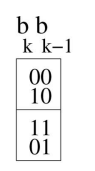

(b)

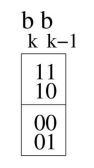

(c)

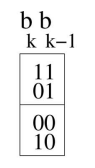

(d)

$$
\begin{array}{|l|}
\begin{array}{l}
\mathrm{b} b \\
\mathrm{k} \mathrm{k}-1
\end{array} \\
\begin{array}{|l|}
\hline 10 \\
01 \\
\hline 00 \\
11 \\
\hline
\end{array}
\end{array}
$$

(e)

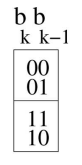

(f)

Fig. 21. Possible combinations of bits $b_{k-1}$ and $b_{k}$ for a pair. The bits used to identify the set are depicted alongside each case. (a) Case 1: $\neg\left(b_{k-1} \oplus b_{k}\right), b_{k-1}$, (b) Case 2: $\neg b_{k-1}, b_{k}$. (c) Case 3: $b_{k}, b_{k-1}$. (d) Case 4: $b_{k-1}, b_{k}$. (e) Case 5: $b_{k-1} \oplus b_{k}, b_{k-1}$. (f) Case 6: $\neg b_{k}, b_{k-1}$.

associativity. Hence, like in IMAP, the four partitions in a group need to be split into two pairs. However, unlike in IMAP, we need to be able to differentiate between the two pairs to indicate which pair gets higher associativity. Therefore, the number of ways of dividing four partitions of a group into two nonidentical sets of two partitions (IPs) each is given by

$$
N_{V-A S S O C}=\frac{4 !}{2 ! 2 !}=6 .
$$

These six Cases are depicted in Fig. 21. To distinguish between the higher and lower associativity pair, we can use $\neg\left(b_{k-1} \oplus b_{k}\right)$ for Case 1, $\neg b_{k-1}$ for Case 2, $b_{k}$ for Case 3, $b_{k-1}$ for Case $4, b_{k-1} \oplus b_{k}$ for Case 5 , and $\neg b_{k}$ for Case 6 . Once the pair to which an address maps is identified, one among the two sets (corresponding to the two entries in a pair) needs to be identified. For this, we can use $b_{k-1}, b_{k}, b_{k-1}, b_{k}$, $b_{k-1}$, and $b_{k-1}$, respectively, for Cases 1 to 6 .

The decision bits of V-ASSOC need to pick one of the six 2 bit pairs of Fig. 21. The lower order bits $\left\langle b_{k-2}, \ldots, b_{1}\right\rangle$ are used to identify the group and pick out four candidate sets (refer to Fig. 22b). Two additional bits are then chosen using three decision bits to identify one among the four candidates. The physical organization for V-ASSOC can be worked out in a similar fashion. We do not detail it here in the interest of space.

\section{Results and Inferences}

This section compares the various two-level mapping-based schemes with existing one-level mapping based functions. Comparisons are made in terms of: 1) the distribution of the useful trie nodes among the cache sets; 2) reduction in number of misses; and 3) memory access time speedup. Although we evaluated the performance of caches of various sizes and associativities, due to space constraints, we only

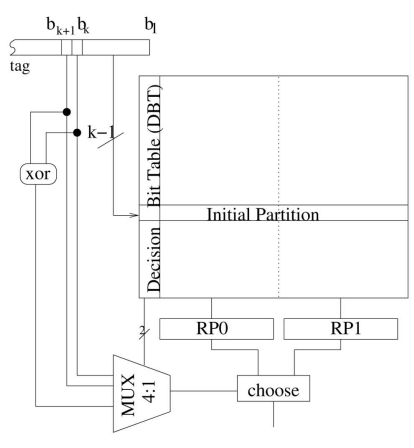

(a)

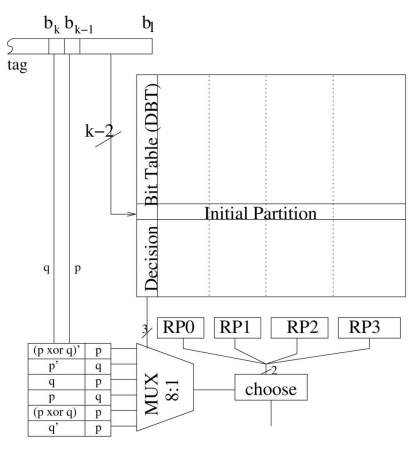

(b)
Fig. 22. Cache architecture for IMAP and V-ASSOC. (a) IMAP. (b) V-ASSOC. report the performance for a cache with $8 \mathrm{k}$ lines for associativities 1, 2, and 4. For V-ASSOC, we simulate a $5 \mathrm{~K}$ line base cache with five-way associativity and compare it with a V-ASSOC cache of the same size with half its sets having associativity 4 and the remaining half with associativity 6 . This ratio of low : high is used because for the routing tables in consideration, the number of nodes mapping to sets with lower associativity is roughly two-third the number of nodes mapping to sets with higher associativity.

\subsection{Node to Set Distribution}

Fig. 23 shows the distribution of nodes to cache sets achieved by conventional mapping, BS, XOR placement, FLEX, IMAP, and V-ASSOC for a cache with 1,024 sets. These plots are for the FUNET routing table. In the case of V-ASSOC, each set has either a high or a low associativity; hence, we plot the average number of nodes mapping to a cache line instead of a cache set. As all lines within a set are equivalent, a fair measure for the number of nodes mapping to a cache line is the number of nodes mapping to a set divided by the associativity of the set. Intuitively, the scheme with a distribution having a narrow spread around the mean value would be best suited for caching. The natural metric to measure this spread would be the coefficient of variation $(\mathrm{CoV})$ of the number of nodes in a cache set (cache line for V-ASSOC). The mean $(\mu)$ and coefficient of variation $\mathrm{CoV}$ are calculated as

$$
\mu=\frac{\text { number_of_useful_nodes }}{N}, C o V=\frac{1}{\mu} \sqrt{\frac{1}{N} \sum_{i=1}^{N}\left(x_{i}-\mu\right)^{2}},
$$

where $N$ is the number of cache sets (cache lines for $\mathrm{V}$-ASSOC) and $x_{i}$ represents the number of nodes mapping to the $i$ th set (line).

The $C o V$ values of the various schemes are shown in Table 9. It can be seen that the XOR-based placement, as it does not make use of the distribution of useful nodes, is unable to improve the node to set distribution. The remaining schemes achieve a lower $C o V$. While this metric helps rank the schemes, only a detailed cache simulation can tell whether they improve performance.

\subsection{Performance of Bit Selection}

In Fig. 24, we plot the percentage misses of bit selection relative to an LO cache with no bit selection. It can be seen that no significant gain is obtained from BS. We find that most of the bits selected are lower order bits that would have been used by the base scheme. The selected index differs from the conventional index in only a few bits (three for the FUNET and two for the Oregon routing table). This explains the lack of a significant performance improvement. 


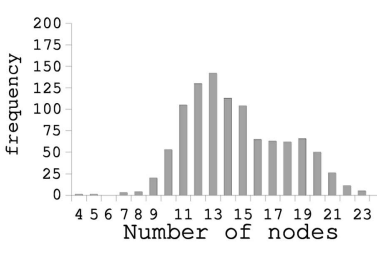

(a)

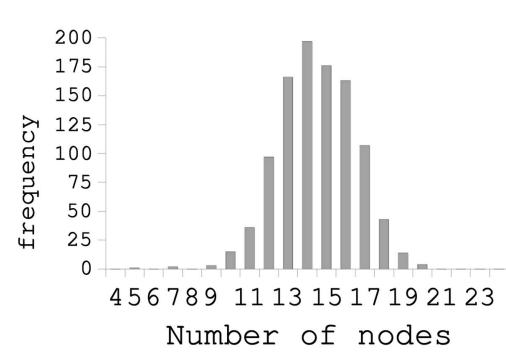

(e)

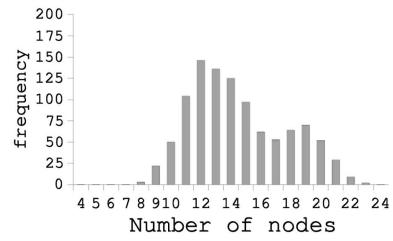

(b)

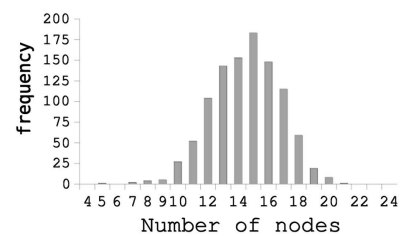

(c)

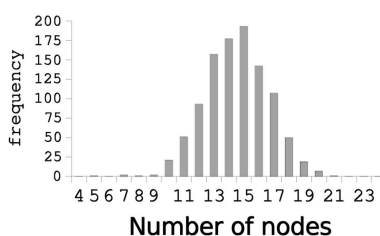

(d)

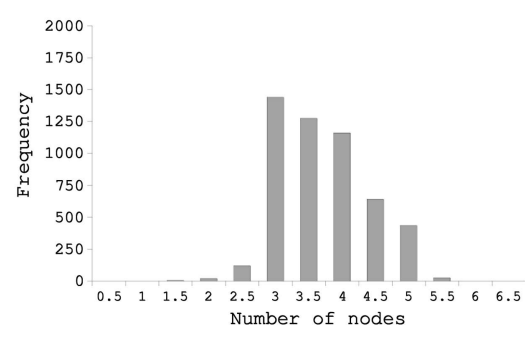

(f)

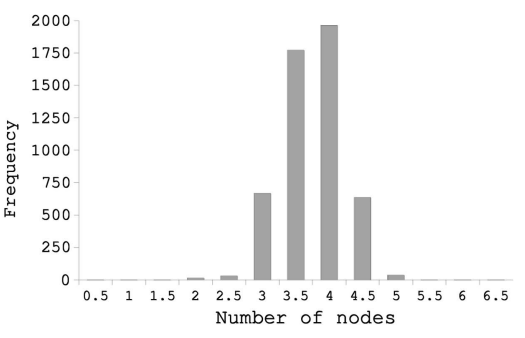

(g)

Fig. 23. Node distribution. y axis represents number of cache sets in (a)-(e) and cache lines in (f) and (g). In (f) and (g) the $x$ axis values represent ranges and not absolute values. For example, 5 refers to the range of values $4.5<x \leq 5$. (a) Conventional Mapping. (b) XOR. (c) BS. (d) FLEX. (e) IMAP. (f) Conventional Mapping. (g) V-ASSOC.

\subsection{Performance of IMAP and FLEX}

Figs. 25 and 26 compare the miss rate reduction and memory access speedup, respectively, for the Oregon routing table. Figs. 27 and 28 compare the same for the FUNET table. The plots in Figs. 25 and 27 show the number of misses incurred by various schemes as a percentage of the misses incurred by the base LO cache. The memory access time speedup is also measured with respect to HSCA with base LO. It is observed that, in comparison to the base scheme, the XORbased scheme performs slightly better for the FUNET route table, while for the Oregon route table, it degrades the performance. Even for configurations where XOR performs better, it at best reduces the number of misses by 3 percent and leads to a speedup in average access time of at most 2 percent.

It is seen that for most configurations, both FLEX and IMAP perform better than the base cache. The additional flexibility in mapping leads to this improvement in performance. Among the two, IMAP performs better for all configurations, reducing the misses by up to 16 percent and speedup in memory acess time of up to 13 percent. Note that the trace for which we achieve the best miss reduction (pld1M) is not the one to attain the best access speedup. This is because of the lower miss rates of the pld1M trace, the trace with maximum locality. This lower miss rate implies that the hit time $(h t)$ becomes more significant, thereby diminishing the contribution of miss rate reduction toward speedup. It can also be observed that, even though the improvement in miss rates fluctuates with

TABLE 9

$\mathrm{CoV}$ Values for Various Schemes

\begin{tabular}{||l|c|c|c|c||}
\hline Scheme & Useful nodes & $N$ & $\mu$ & $C o V$ \\
\hline Base LO & 14859 & 1024 & 14.51 & 0.2251 \\
\hline XOR & 14859 & 1024 & 14.51 & 0.2204 \\
\hline BS & 14859 & 1024 & 14.51 & 0.1534 \\
\hline FLEX & 14859 & 1024 & 14.51 & 0.1464 \\
\hline IMAP & 14859 & 1024 & 14.51 & 0.1375 \\
\hline V-ASSOC & 14859 & 5120 & 2.90 & 0.1536 \\
\hline
\end{tabular}

change in locality, the memory access time speedups achieved are more or less consistent. Finally, it is to be noted that it is unlikely that in any scenario, either FLEX or IMAP will degrade performance drastically. This is because both FLEX and IMAP have the choice of picking the base set of index bits if they find nothing better. The overall memory access time speedup obtained with FLEX and IMAP in comparison to unified cache are plotted in Fig. 29. With IMAP, the memory access time can be improved by up to 40 percent. This is an additional 15 percent above what base HSCA can achieve and is gained under traffic with worstcase locality.

\subsection{Performance of V-ASSOC}

Comparison of V-ASSOC with the base cache (refer to Fig. 30) shows that V-ASSOC degrades the performance for all traces. It is interesting to note that the CoV of V-ASSOC is slightly higher than that for BS (Table 9). As BS does not provide significant benefits, it is not surprising that V-ASSOC does not either.

\subsection{Timing Analysis}

We model the placement function part of the cache (both for tag and data array) in detail using HSPICE for $90 \mathrm{~nm}$ technology. We only report the timing analysis for FLEX and IMAP as they are the better performing schemes. The

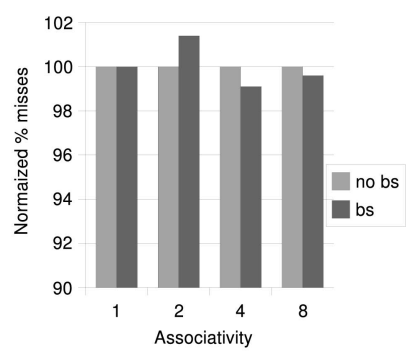

(a)

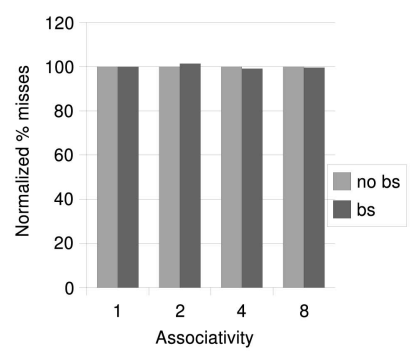

(b)
Fig. 24. Impact of BS, FUNET. (a) 4K. (b) $8 \mathrm{~K}$. 


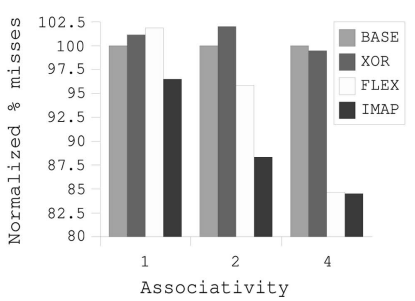

(a)

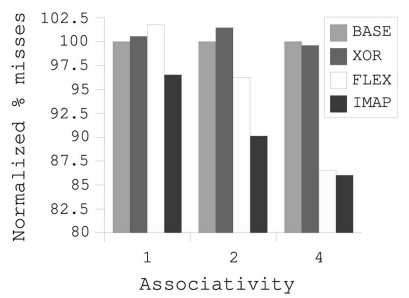

(b)

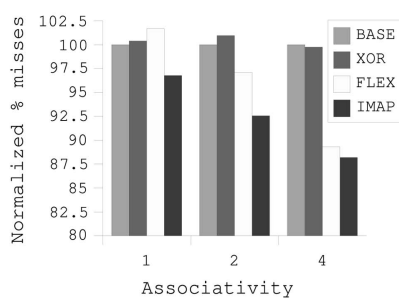

(c)

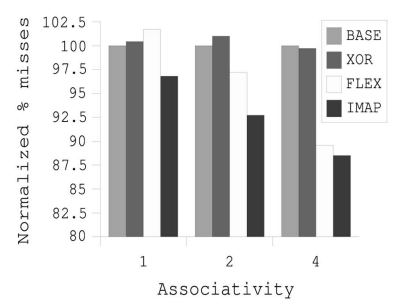

(d)

Fig. 25. Miss rate comparisons for the Oregon route table. (a) pld1M. (b) pld10M. (c) pld100M. (d) pld1B.

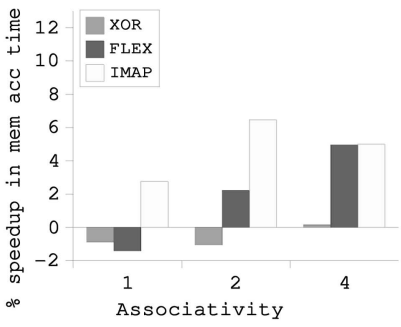

(a)

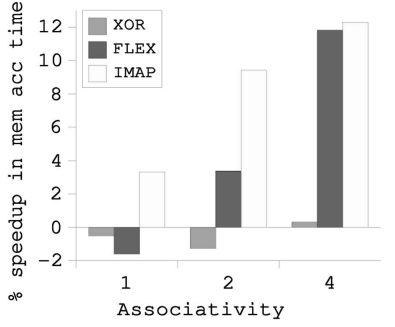

(b)

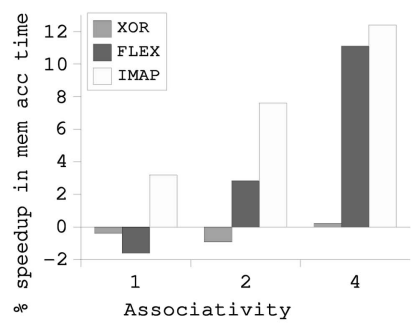

(c)

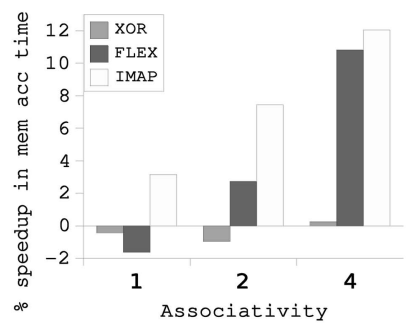

(d)

Fig. 26. Average memory access time comparisons for the Oregon route table. (a) pld1M. (b) pld10M. (c) pld100M. (d) pld1B.

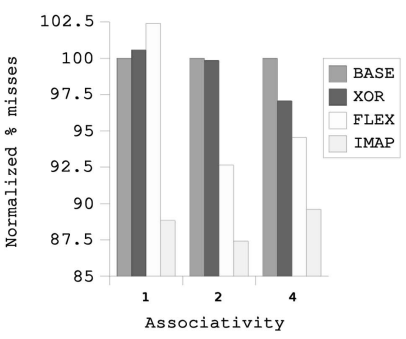

(a)

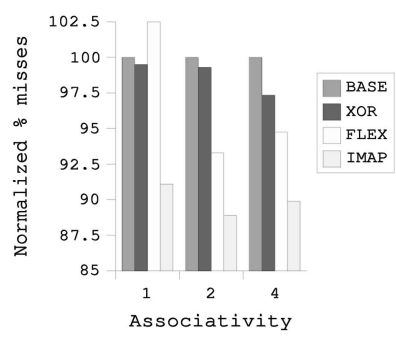

(b)

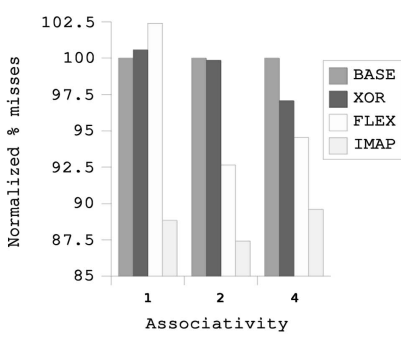

(c)

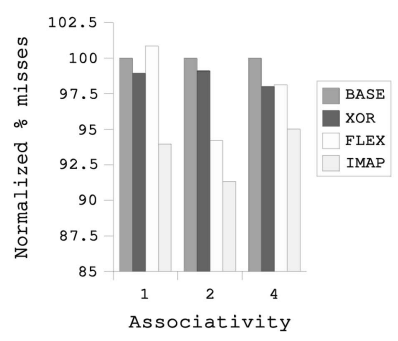

(d)

Fig. 27. Miss rate comparisons for the FUNET route table. (a) pld1M. (b) pld10M. (c) pld100M. (d) pldB.

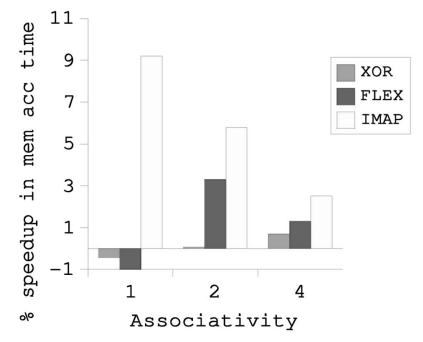

(a)

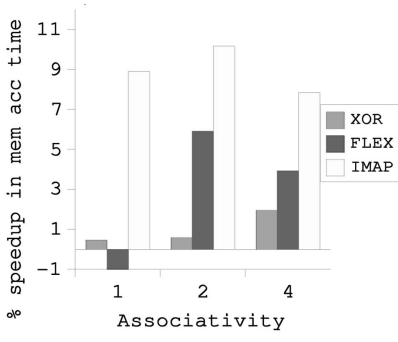

(b)

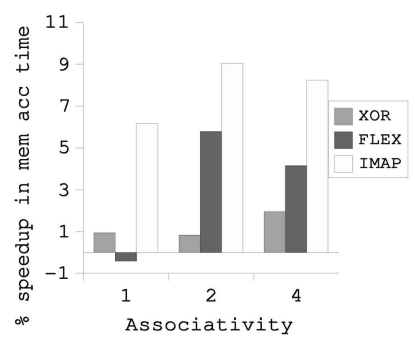

(c)

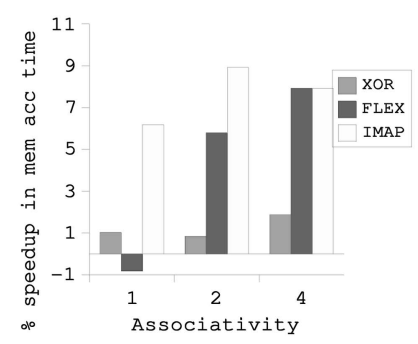

(d)

Fig. 28. Average memory access time comparisons for FUNET route table. (a) pld1M. (b) pld10M. (c) pld100M. (d) pld1B.

two possible critical paths for an FLEX- or IMAP-based cache are shown in Fig. 31. The conventional critical path, refered to as the row path, is marked in blue and has a delay $d_{4}$ associated with it. The other possible critical path through the column decoders (col path) is shown in red and has a delay of $d_{1}+d_{2}+d_{3}$. Our spice model uses circuits for decoders as suggested by CACTI [38], [33]. The delay of the decoders depend not only on the logic for decoding but also the load being driven. For the row decoder, the (wordline) driver delay depends on the length of the wordline and the number of bit cells along the wordline. For the column decoder, it depends on the number of column multiplexers (nmos pass transistors), it is driving and the length of the wires. The base cache organization (both data and tag part) including the number and size subarrays and extent of column multiplexing are estimated using CACTI [33].

Table 10a shows the breakdown of delays incurred by the components of the two paths for an $8 \mathrm{~K}$ line 2 way associative LO cache. As can be seen the time taken across both the paths is almost identical. The delay introduced to accomplish remapping is well hidden by the organization. The delay for direct mapped, two-way and four-way associative caches of sizes $4 \mathrm{~K}$ and $8 \mathrm{~K}$ lines are shown in Table $10 \mathrm{~b}$. We also report the fraction of increase in cache access time assuming the additional delay introduced by two-level mapping to be $\max \left(\operatorname{tag}_{\text {extra }}\right.$, data $\left._{\text {extra }}\right)$. The base access times are obtained from CACTI. As can be seen, the increase in cache access times is negligible $(<4.5$ percent) with a maximum increase 


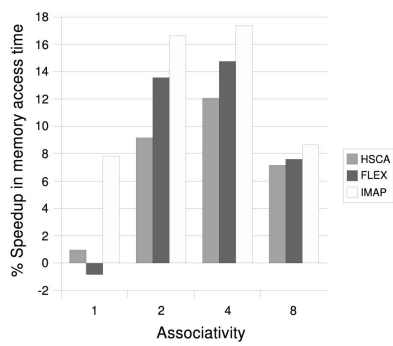

(a)

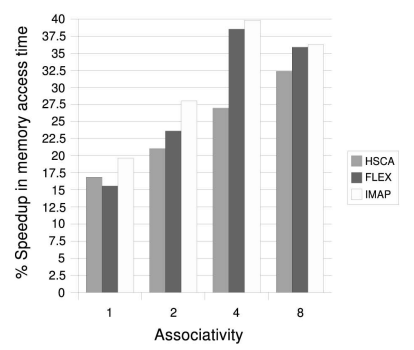

(b)
Fig. 29. Overall speedup in memory access time with two-level mapping. (a) Speedup in memory access time, FUNET, pld10M. (b) Speedup in memory access time, Oregon, pld1B.

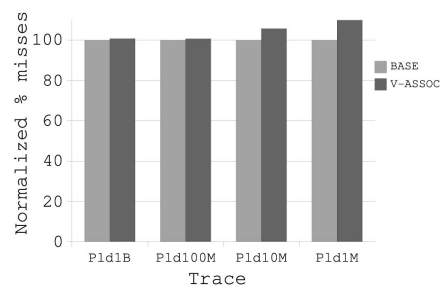

Fig. 30. Miss rate comparison for V-ASSOC.

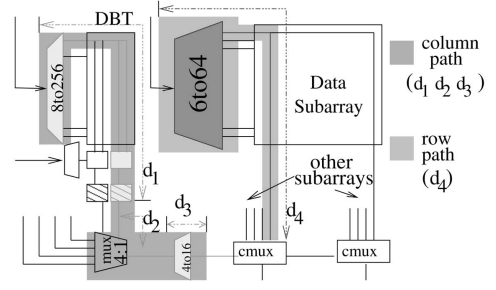

Fig. 31. Placement function-critical paths.

TABLE 10

Cache Access Time

(a) Component Delays and (b) Access Time Increase

\begin{tabular}{||l|l|r|r||}
\hline \multicolumn{2}{||c|}{ component } & data(ps) & tag(ps) \\
\hline \multirow{3}{*}{$\begin{array}{l}\text { column } \\
\text { path }\end{array}$} & DBT $\left(d_{1}\right)$ & 137.7 & 131.1 \\
\cline { 2 - 4 } & $4: 1$ mux $\left(d_{2}\right)$ & 31.3 & 31.3 \\
\cline { 2 - 4 } & decoder $\left(d_{3}\right)$ & 54.3 & 62.2 \\
\cline { 2 - 4 } & tot $\left(d_{1}+d_{2}+d_{3}\right)$ & 223.3 & 224.6 \\
\hline row path & sub-array $\left(d_{4}\right)$ & 222.9 & 210.7 \\
\hline \multicolumn{2}{||}{ data daxtra $_{\text {extag }}$, tag $_{\text {extra }}($ col - row $)$} & 0.4 & 13.9 \\
\hline
\end{tabular}

(a)

\begin{tabular}{||l|r|r|r|}
\hline Configuration & Extra & Acc time & $\%$ extra \\
\hline 8K line dm & $23.8 \mathrm{ps}$ & $633.6 \mathrm{ps}$ & 3.7 \\
8K line 2 way & $13.9 \mathrm{ps}$ & $673.7 \mathrm{ps}$ & 2.0 \\
8K line 4 way & $12.4 \mathrm{ps}$ & $637.3 \mathrm{ps}$ & 1.9 \\
4K line dm & $24.9 \mathrm{ps}$ & $550.3 \mathrm{ps}$ & 4.5 \\
4K line 2 & $19.8 \mathrm{ps}$ & $604.7 \mathrm{ps}$ & 3.2 \\
4K line 4 way & $0 \mathrm{ps}$ & $609.4 \mathrm{ps}$ & 0 \\
\hline
\end{tabular}

(b)

of 25 ps and should not impact cycle time especially at the lower frequencies with which NPs operate.

\section{Conclusions}

This paper makes the following contributions:

- An attribute preserving trace generation methodology is proposed to generate synthetic traces with prefix length distribution and locality characteristics of real traces.

- A heterogeneously segmented cache architecture is proposed to take advantage of the differences in locality characteristics at various levels of the trie.

- A weight-based replacement policy is proposed that is effective at reducing misses on core routers.

- A two-level mapping framework is proposed to reduce conflict misses in the Level-One nodes cache of HSCA.

- Three low overhead remapping functions (FLEX, IMAP, and V-ASSOC) are proposed to facilitate twolevel mapping and improve the node to set distribution.

- Modifications to the cache organization are proposed and a detailed timing analysis based on HSPICE simulations is performed to ensure that the remap can be accommodated with minimal increase in access time.

As future work, we plan to evaluate the benefits of HSCA in a multiprocessor environment. We also plan to extend two-level mapping framework to general purpose processor caches.

\section{References}

[1] B. Agarwal and T. Sherwood, "Virtually Pipelined Network Memory," Proc. Int'l Symp. Microarchitecture, 2006.

[2] J.-L. Baer, D. Low, P. Crowley, and N. Sidhwaney, "Memory Hierarchy Design for a Multiprocessor Look-Up Engine," Proc. Int'l Conf. Parallel Architectures and Compilation Techniques, 2003.

[3] A. Brodnik, S. Carlsson, M. Degermark, and S. Pink, "Small Forwarding Tables for Fast Routing Lookups," Proc. ACM SIGCOMM '97, 1997.

[4] H.J. Chao, "Next Generation Routers," Proc. IEEE (Invited Paper), vol. 90, no. 9, pp. 1518-1558, 2002.

[5] T. Chieueh and K. Gopalan, "Improving Route Lookup Performance Using Network Processor Cache," IEEE/ACM Supercomputing Conf., 2002.

[6] T. Chieueh and P. Pradhan, "Cache Memory Design for Network Processors," Proc. Int'l Symp. High Performance Computer Architecture, 2000.

[7] K.C. Claffy, "Internet Traffic Characterization," PhD thesis, Univ. of California, San Diego, 1994.

[8] J. Edler and M.D. Hill, "Dinero IV Trace-Driven Uniprocessor Cache Simulator," www.cs.wisc.edu/markhill/DineroIV/, 1998.

[9] T. Givargis, "Improved Indexing for Cache Miss Reduction in Embedded Systems," Proc. Design Automation Conf. (DAC), 2003.

[10] A. González, M. Valero, N. Topham, and J.M. Parcerisa, "Eliminating Cache Conflict Misses through XOR-based Placement Functions," Proc. Int'l Conf. Supercomputing, 1997.

[11] E.G. Hallnor and S.K. Reinhardt, "A Fully Associative SoftwareManaged Cache Design," Proc. Int'l Symp. Computer Architecture, 2000.

[12] P. Gupta, S. Lin, and N. McKeown, "Routing Lookups in Hardware at Memory Access Speed," Proc. IEEE Infocom, 1998.

[13] J. Hasan, S. Chandra, and T.N. Vijaykumar, "Efficient Use of Memory Bandwidth to Improve Network Processor Throughput," Proc. Int'l Symp. Computer Architecture, 2003.

[14] J.L. Hennessey and D.A. Patterson, Computer Architecture: A Quantitative Approach, third ed. Morgan Kaufmann Publishers, Inc., 2003.

[15] R. Jain, "Characteristics of Destination Address Locality in Computer Networks: A Comparison of Caching Schemes," Computer Networks and ISDN Systems, 1990.

[16] M. Kharbutli, K. Irwin, Y. Solilin, and J. Lee, "Using Prime Numbers for Cache Indexing to Eliminate Conflict Misses," Proc. IEEE Int'l Symp. High Performance Computer Architecture, 2004.

[17] B. Lampson, V. Srinivasan, and G. Varghese, "IP Lookup Using Multiway and Multicolumn Search," Proc. IEEE Infocom, 1998.

[18] H. Liu, "Routing Prefix Caching in Network Processor Design," Proc. Int'l Conf. Computer Comm. and Networks, 2001. 
[19] H. Liu, "Reducing Cache Miss Ratio For Routing Prefix Cache," Proc. Global Telecomm. Conf. (GLOBECOM), 2002.

[20] D.R. Morrison, "PATRICIA-Practical Algorithm to Retrieve Information Coded in Alphanumeric," J. ACM, 1968.

[21] J. Mudigonda, H.M. Vin, and R. Yavatkar, "Overcoming the Memory Wall in Packet Processing: Hammers or Ladders?," Proc. Int'l Symp. Architectures for Networking and Comm. System (ANCS), 2005.

[22] G. Narlikar and F. Zane, "Performance Modeling for Fast IP Lookups," Proc. ACM SIGMETRICS, 2001.

[23] S. Nilsson and G. Karlsson, "IP-address Lookup Using LC-Tries," IEEE J. Selected Areas in Comm., vol. 17, no. 6, June 1999.

[24] M.K. Qureshi, D. Thompson, and Y.N. Patt, "The V-Way Cache: Demand Based Associativity via Global Replacement," Proc. Int'l Symp. Computer Architecture, 2005.

[25] K. Rajan and R. Govindarajan, "A Heterogeneously Segmented Cache Architecture for a Packet Forwarding Engine," Proc. Int'l Conf. Supercomputing, 2005.

[26] K. Rajan and R. Govindarajan, "Two-Level Mapping Based Cache Index Selection for Packet Forwarding Engines," Proc. Int'l Conf. Parallel Architectures and Compilation Techniques, 2006.

[27] V.C. Ravikumar, R. Mahapatra, and J.C. Liu, "Modified LC-Trie Based Efficient Routing Lookup," Proc. IEEE Int'l Symp. Modeling, Analysis, and Simulation of Computer and Telecomm. Systems (MASCOTS '02), 2002.

[28] J. Rooney, J. Delgado Frias, and D. Summerville, "An Associative Ternary Cache for IP Routing," Proc. Computers and Digital Techniques, 2004.

[29] M. Ruiz-Sanchez et al., "Survey and Taxonomy of IP Address Lookup Algorithms," IEEE Network Magazine, vol. 15, no. 2, Mar./ Apr. 2001

[30] G. Shenoy and R. Govindarajan, "Performance Modeling and Architecture Exploration of Network Processors," Proc. Int'l Conf. Quantitative Evaluation of Systems (QEST), 2005.

[31] T. Sherwood, G. Varghese, and B. Calder, "A Pipelined Memory Architecture for High Throughput Network Processors," Proc. Int'l Symp. Computer Architecture, 2003.

[32] W. Shi et al., "Synthetic Trace Generation for Internet," Proc. IEEE Workshop Workload Characterization (WWC-4), 2001.

[33] P. Shivakumar and N.P. Jouppi, "CACTI 3.0: An Integrated Cache Timing, Power, and Area Model," DEC WRL Research, 2001.

[34] D. Thiebaut et al., "Synthetic Traces for Trace-Driven Simulation of Cache Memories," IEEE Trans. Computers, vol. 41, no. 4, pp. 388410 Apr. 1992.

[35] V. Srinivasan and G. Varghese, "Fast Address Lookups Using Controlled Prefix Expansion," ACM Trans. Computer Systems, 1999.

[36] B. Talbot, T. Sherwood, and B. Lin, "IP Caching for Terabit Speed Routers," Proc. IEEE Globcom, 1999.

[37] J. Verdu, J. Garcia, M. Nemirovsky, and M. Valero, "Architectural Impact of Stateful Networking Applications," Proc. Int'l Symp. Architectures for Networking and Comm. Systems (ANCS), 2005.

[38] M. Waldvogel, G. Varghese, J. Turner, and B. Plattner, "Scalable High Speed IP Routing Lookup," Proc. ACM SIGMETRICS, 2001.

[39] S.J.E. Wilton and N.P. Jouppi, "CACTI: An Enhanced Cache Access and Cycle Time Model," IEEE J. Solid-State Circuits, vol. 31, no. 5, May 1996.

[40] http://www.nadh.kth.se/ snilsson, 2007.

[41] Univ. of Oregon Route Views Project, www.routeviews.org, 2009.

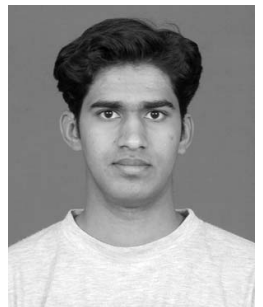

Kaushik Rajan received the BTech degree in information technology from the University of Madras in 2003 and the $\mathrm{PhD}$ degree from the Supercomputer Education and Research Centre, Indian Institute of Science, Bangalore, India, in 2008 . He is currently a postdoctoral researcher with the Rigorous Software Engineering Group at Microsoft Research, Bangalore, India. His research interest is in computer systems with focus on computer architecture, static analysis, performance modeling, and programming languages.

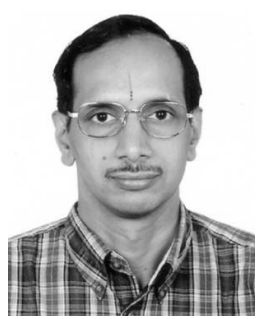

R. Govindarajan received the BSc degree in mathematics from Madras University in 1981, and the BE degree in electronics and communication, and the $\mathrm{PhD}$ degree in computer science from the Indian Institute of Science, Bangalore, in 1984 and 1989, respectively. He has held postdoctoral research positions at the University of Western Ontario, Canada, and McGill University, Canada, and faculty position in the Department of Computer Science, Memorial University of Newfoundland, Canada. He has held visiting faculty positions at the University of Delaware and Arizona State University. Since 1995, he has been with the Supercomputer Education and Research Centre and the Department of Computer Science and Automation, Indian Institute of Science, where he is currently a professor and the chairman of the Supercomputer Education and Research Centre. $\mathrm{He}$ is a senior member of the IEEE and the IEEE Computer Society.

$\triangleright$ For more information on this or any other computing topic, please visit our Digital Library at www.computer.org/publications/dlib. 